\title{
Magnetic, orbital and charge ordering in the electron-doped manganites
}

\author{
Tulika Maitra*1 and A. Taraphder ${ }^{\dagger 2}$ \\ *Max-Planck-Institut für Physik Komplexer Systeme \\ Nöthnitzer Str. 3801187 Dresden, Germany \\ ${ }^{\dagger}$ Department of Physics \& Meteorology and Centre for Theoretical Studies, \\ Indian Institute of Technology, Kharagpur 721302 India
}

\begin{abstract}
The three dimensional perovskite manganites $\mathrm{R}_{1-x} \mathrm{~A}_{x} \mathrm{MnO}_{3}$ in the range of holedoping $x>0.5$ are studied in detail using a double exchange model with degenerate $e_{g}$ orbitals including intra- and inter-orbital correlations and near-neighbour Coulomb repulsion. We show that such a model captures the observed phase diagram and orbital-ordering in the intermediate to large band-width regime. It is argued that the Jahn-Teller effect, considered to be crucial for the region $x<0.5$, does not play a major role in this region, particularly for systems with moderate to large band-width. The anisotropic hopping across the degenerate $e_{g}$ orbitals are crucial in understanding the ground state phases of this region, an observation emphasized earlier by Brink and Khomskii. Based on calculations using a realistic limit of finite Hund's coupling, we show that the inclusion of interactions stabilizes the C-phase, the antiferromagnetic metallic A-phase moves closer to $x=0.5$ while the ferromagnetic phase shrinks in agreement with recent observations. The charge ordering close to $x=0.5$ and the effect of reduction of band-width are also outlined. The effect of disorder and the possibility of inhomogeneous mixture of competing states have been discussed.
\end{abstract}

PACS Nos. 75.30.Et, 75.47.Lx, 75.47.Gk

\section{Introduction}

The colossal magnetoresistive manganites have been investigated with renewed vigour in the recent past mainly because of their technological import. It was soon realized that these systems have a rich variety of unusual electronic and magnetic properties involving almost all the known degrees of freedom in a solid, viz., the charge, spin, orbital and lattice degrees of freedom[1,2,3]. Of particular interest have been the systems $\mathrm{R}_{1-x} \mathrm{~A}_{x} \mathrm{MnO}_{3}$, where $R$

\footnotetext{
${ }^{1}$ email: tulika@mpipks-dresden.mpg.de

${ }^{2}$ email: arghya@phy.iitkgp.ernet.in, arghya@cts.iitkgp.ernet.in
} 
and $A$ stand for trivalent rare-earth (e.g., La, Nd, $\mathrm{Pr}, \mathrm{Sm}$ ) and divalent alkaline-earth ions (Ca, $\mathrm{Sr}, \mathrm{Ba}, \mathrm{Pb}$ etc.) respectively. Around the region $0.17<x<0.4$, electrical transport properties of these systems generically show extreme sensitivity towards external magnetic field with a concomitant paramagnetic insulator (or poor metal) to ferromagnetic metal transition at fairly high temperatures $[5,6,7]$. For a long time the dominant paradigm in the theory of this unusual magnetic field-dependence of transport has been the idea of double exchange (DE) [8] involving the localized core spins (the three $t_{2 g}$ electrons at each Mn site) coupled to the itinerant electrons in the Jahn-Teller split $e_{g}$ level via strong Hund's exchange. It has been realized recently that such a simplifying theoretical framework may not be adequate in explaining several other related features involving transport, electronic and magnetic properties $[9,10,11,12,13]$. It was already known that the observed structural distortions and magnetic and orbital orders in these systems in the region $x \simeq 0.5$ require interactions not included in the DE model $[14,15,16]$.

Owing to the observation of colossal magnetoresistance (CMR) in the region $x<0.5$ in the relatively narrow band-width materials [6] at high temperatures, much of the attention was centred around this region. Only in the last few years CMR effect has been observed in the larger band-width materials like $\mathrm{Nd}_{1-x} \mathrm{Sr}_{x} \mathrm{MnO}_{3}[17,18]$ and $\mathrm{Pr}_{1-x} \mathrm{Sr}_{x} \mathrm{MnO}_{3}[19,20]$ in the region $x>0.5$. If one counts the doping from the side $x=1$ in $\mathrm{R}_{1-x} \mathrm{~A}_{x} \mathrm{MnO}_{3}$ where all $\mathrm{Mn}$ ions are in +4 state, then doping by $R_{y}(y=1-x)$ introduces $\mathrm{Mn}^{+3}$ ions carrying one electron in the $e_{g}$ orbitals. This region, therefore, is also called the electron-doped region. The charge, magnetic and orbital structures of the manganites in the electron-doped regime have already been found to be quite rich $[2,4,21,22,23]$ and the coupling between all these degrees lead to stimulating physics [24].

In the framework of the conventional DE model with one $e_{g}$ orbital, one would expect qualitatively similar physics for $x \sim 0$ and $x \sim 1$ [25]. On the contrary, experiments reveal a very different and assymetric picture for the phase diagram between the regions $x<0.5$ and 
$x>0.5$. The lack of symmetry about $x=0.5$ manifests itself most clearly in the magnetic phase diagram of these manganites. It has now been shown quite distinctly $[18,32,27,34]$ that the systems $\mathrm{Nd}_{1-x} \mathrm{Sr}_{x} \mathrm{MnO}_{3}, \mathrm{Pr}_{1-x} \mathrm{Sr}_{x} \mathrm{MnO}_{3}, \mathrm{La}_{1-x} \mathrm{Sr}_{x} \mathrm{MnO}_{3}$ are antiferromagnetically ordered beyond $x=0.5$ while one observes either a metallic ferromagnetic state [7] or a charge ordered state with staggered charge-ordering $[29,30]$ in the approximate range $0.25<x<0.5$. This charge ordered insulating state can be transformed into a ferromagnetic metallic state $[19,31]$ by the application of magnetic fields.

There are several different types of AFM phases with their characteristic dimensionality of spin ordering observed in this regime. $\mathrm{La}_{1-x} \mathrm{Sr}_{x} \mathrm{MnO}_{3}$ shows A-type antiferromagnetic ground state (in which ferromagnetically aligned $x y$-planes are coupled antiferromagnetically) in the range $0.52<x<0.58$. It also shows a sliver of FM phase [27] immediately above $x=0.5$. In $\mathrm{Nd}_{1-x} \mathrm{Sr}_{x} \mathrm{MnO}_{3}[34,18]$, the A-type spin structure appears at $x=0.5$ and is stable upto $x=0.62$ while in $\operatorname{Pr}_{1-x} \mathrm{Sr}_{x} \mathrm{MnO}_{3}[19,26]$, this region extends from $x=0.48$ to $x=0.6$. In all these cases, the phase that abuts the A-type antiferromagnet (AFM) in the region of higher hole-doping $(x)$ is the C-type AFM state, in which antiferromagnetically aligned planes are coupled ferromagnetically. The C-type AFM phase occupies largest part of the phase diagram in this region. For even larger $x$, the $\mathrm{C}$-phase gives way to the three dimensional antiferromagnetic G-phase.

The systematics of the phase diagram changes considerably (except close to $x=1$ ) in these systems as a function of the bandwidth. Recently Kajimoto et al. [28] have quite succinctly summarized the phase diagrams of various manganites of varying bandwidths across the entire range of doping. Their phase diagram is reproduced in fig. 1. The phase diagram changes considerably with changing bandwidth as shown in the figure. We note that the narrow bandwidth compounds like $\mathrm{Pr}_{1-x} \mathrm{Ca}_{x} \mathrm{MnO}_{3}, \mathrm{La}_{1-x} \mathrm{Ca}_{x} \mathrm{MnO}_{3}$ etc. exhibit a wide region of CE-type insulating charge-ordered state around $x=0.5$ whereas the intermediate bandwidth material $\mathrm{Nd}_{1-x} \mathrm{Sr}_{x} \mathrm{MnO}_{3}$ shows a conducting A-type antiferromagnetic 
phase around $x=0.5$. As one moves towards the larger bandwidth compounds such as $\operatorname{Pr}_{1-x} \mathrm{Sr}_{x} \mathrm{MnO}_{3}, \mathrm{La}_{1-x} \mathrm{Sr}_{x} \mathrm{MnO}_{3}$, a small strip of ferromagnetic (F) metallic phase appears at $x=0.5[27,28]$ followed by the A-type AFM state. In contrast with the narrow bandwidth manganites, the relatively wider bandwidth manganites generally show the following sequence of spin/charge ordering upon hole doping (in the entire range $0 \leq x \leq 1$ ): insulating A-type AFM $\rightarrow$ metallic FM $\rightarrow$ metallic A-type AFM $\rightarrow$ insulating C-type AFM and finally insulating G-type AFM states. Clearly, the most important feature here is the absence of CE-type spin/charge ordering and the presence of a metallic A-type AFM state in these wider band-width compounds in the region close to $x=0.5$. It appears that the physics involved in the CE-type charge/spin ordering, important for the low band-width systems, is not quite as relevant in this case. In addition, it is also observed in the neutron diffraction studies that the metallic A-type AFM state is orbitally ordered $[32,34]$ with predominant occupation of $d_{x^{2}-y^{2}}$ orbitals. The importance of orbital-ordering has been emphasized previously in several other experimental $[18,35,39,40]$ and theoretical $[33,41,42,43,44,45,46]$ investigations. The crucial role of the $e_{g}$ orbitals and inter-orbital Coulomb interaction has been underlined by Takahashi and Shiba [50] from a study of the optical absorption spectra in the ferromagnetic metallic phase of the doped manganite $\mathrm{La}_{1-x} \mathrm{Sr}_{x} \mathrm{MnO}_{3}$. They point out that it is imperative to consider the transition between nearly degenerate and moderately interacting $e_{g}$ orbitals even in the hole-doped region in order to interpret the optical absorption spectra in $\mathrm{La}_{1-x} \mathrm{Sr}_{x} \mathrm{MnO}_{3}$.

In a detailed observation carried out by Akimoto et al. [27] the electronic and magnetic properties of a heavily doped manganite $R_{1-x} S r_{x} M n O_{3}$ with $R=L a_{1-z} N d_{z}$ are studied by continuously changing the band-width. In this novel procedure they were able to control the band-width chemically by changing the average ionic radius by manipulating the ratio of La and Nd (i.e., changing $z$ ). Substitution of the smaller $N d^{+3}$ ions for the larger $\mathrm{La}^{+3}$ ions effectively reduces the one-electron band-width. By increasing $z$ chemically, they were 
able to go continuously from the large band-width system $\mathrm{La}_{1-x} \mathrm{Sr}_{x} \mathrm{MnO}_{3}$ down to the intermediate band-width system $N_{1-x} S_{x} M n O_{3}$. For $z<0.5$, there is a metallic FM phase in the region $0.5<x<0.52$. From $x \geq 0.54$ to about $x=0.58$ the ground state is A-type antiferromagnetic metallic irrespective of the value of $z$, i.e. from $\mathrm{La}_{1-x} \mathrm{Sr}_{x} \mathrm{MnO}_{3}$ $(z=0)$ all the way down to $N d_{1-x} S_{x} M n O_{3}(z=1)$. They believe that the key factor that stabilizes the A-type AFM metallic state in a wide range of $z$ is the structure of the two $e_{g}$ orbitals $\left(d_{x^{2}-y^{2}}\right.$ and $\left.d_{3 z^{2}-r^{2}}\right)$ and the anisotropic hopping integral between them. There is no signature of charge-ordering or CE-type ordering below $z=0.5$ for any $x$. The chargeordered $(\mathrm{CO})$ insulating state appears above $z=0.5$ and around $x=0.5$ primarily due to the commensuration (between the lattice periodicity and hole concentration) effect in the low band-width systems. The ground state phase diagram for doped manganites in $x-z$ plane (i.e., doping versus band-width plane) is shown in fig. 2 after Akimoto et al. [27].

The general inferences from all these measurements are that the physics of the electrondoped region is very different from the hole-doped region. In this region, with decreasing band-width starting from $\mathrm{La}_{1-x} \mathrm{Sr}_{x} \mathrm{MnO}_{3}$ down to $\mathrm{Nd}_{1-x} \mathrm{Sr}_{x} \mathrm{MnO}_{3}$, the F-phase shrinks, the A-phase and C-phase remain nearly unaffected. The A-phase disappears and the C-phase shrinks (with the possible growth of incommesurate charge order region as in fig. 1) rapidly in the low band-width systems like $\mathrm{La}_{1-x} \mathrm{Ca}_{x} \mathrm{MnO}_{3}$ and $\mathrm{Pr}_{1-x} \mathrm{Ca}_{x} \mathrm{MnO}_{3}$. The G-phase at the low electron-doping region seems to remain unaffected all through. It has been seen $[27,34,35]$ that the gradual build up of the AFM correlations in the electron-doped region is pre-empted by the orbital ordering in the A- and C-phases. The $e_{g}$ orbitals and the anisotropic hopping of electrons between them [16, 63], must indeed play a significant role given the presence of orbital ordering in much of the phase diagram beyond $x=0.5$. It is also realized that the effect of lattice could be ignored in the first approximation for these moderate to large band-width systems in this region of doping. All these point to the fact that the interactions that play a dominant role in the elctron-doped region are different 
$[36,37,38]$ from the ones that are considered crucial in the hole-doped side.

There has been a large number of reports of charge ordering and inhomogeneous states $[17,18,19,47,48,49,52,53]$ in the region $x \simeq 0.5$. These states are quite abundant in the low band-width materials. The inhomogeneous states result primarily from the competing ground states [54] (charge ordered/AFM and FM in this case) that lead to 1st. order phase transitions with a discontinuity in the density as the chemical potential is varied. Such transitions are known to lead to phase separation in the canonical ensemble. Phase separation in this context has been dicussed in the literature for quite some time [51, 55, 56, 57, 58,61]. Such macroscopic phase separations are not stable against long range Coulomb interactions and tend to break up into microscopic inhomogeneities $[55,59,60]$. There is also the wellknown CE-type charge and spin ordering that has been seen at $x=0.5$ in most of these systems $[14,15,47]$ with low band-width.

In both $\mathrm{Nd}_{1-x} \mathrm{Sr}_{x} \mathrm{MnO}_{3}$ and $\mathrm{Pr}_{1-x} \mathrm{Sr}_{x} \mathrm{MnO}_{3}$ Kawano et al. [32] and Kajimoto et al. $[28,34]$ have seen finite temperature $(T \simeq 150 K)$ first order transitions at $x=0.5$ from a ferromagnetic metal to an antiferromagnetic A-phase which is insulating but has quite low resistivity (immediately away from $x=0.5$ it becomes A-type metal). In a neutron diffraction study Kajimoto et al. [28] have also observed that close to the boundary of the FM and A-type AFM metallic phases of $\mathrm{Pr}_{1-x} \mathrm{Sr}_{x} \mathrm{MnO}_{3}$, an unusual stripe-like charge-order appears along with this weakly first order transition.

This stripe-like charge-order is distinctly different from the staggered charge-ordering of the CE-type state. Very recently, an inhomegeneous mixture of micron-size antiferromagnetic grains (possibly charge-ordered) and similar sized ferromagnetic grains has been seen in electron diffraction and dark-field imaging in the low band-width system $\mathrm{La}_{1-x} \mathrm{Ca}_{x} \mathrm{MnO}_{3}$ at $x=0.5[62]$ without any evidence of the long-range $\mathrm{CE}$ or any other macroscopic ordering. The ground state energies of these different phases seem to be very close [67] in this region leading to a possible first order phase transition and consequent phase seggregation. A 
possible nanoscale phase separation between A-type AFM and ferromagnetic regions has recently been reported by Jirac et al., [81] in the cintered ceramic samples of $\operatorname{Pr}_{0.44} \mathrm{Sr}_{56} \mathrm{MnO}_{3}$ doped with $\mathrm{Cr}$ (upto 8 percent). It is also observed that although both the ferromagnetic domains and A-type AFM host are independently metallic (though anisotropic for A-AFM), the resultant inhomohgeneous state is non-metallic.

Almost all the experiments discussed above consider orbital ordering as the underlying reason for the various magnetic orders observed in the electron-doped regime. The anisotropy of the two $e_{g}$ orbitals and the nature of overlap integral between them $[16,63]$ make the electronic bands low dimensional. Such anisotropic conduction in turn leads to anisotropic spin exchanges and different magnetic structures. In the A-phase the kinetic energy gain of the electrons is maximum when the orbitals form a $2 \mathrm{D}$ band in the $x y$-plane and maximize the in-plane ferromagnetic exchange interaction. However, in the $z$-direction AFM superexchange interaction dominates due to the negligible overlap of $d_{x^{2}-y^{2}}$ and $d_{3 z^{2}-r^{2}}$ orbitals. In addition, the presence of charge ordering and inhomegeneous or phase separated states, particularly around the commensurate densities, are suggestive of the vital role of Coulomb interactions in the manganites. The absence of CE-phase in the moderate to large bandwidth materials imply that the role of Jahn-Teller or static lattice distortions may not be as crucial in the electron-doped regime even in the region close to $x=0.5$. A model, for the electron-doped systems, therefore, should have as its primary ingredients, the two $e_{g}$ orbitals at each Mn site and the anisotropy of hopping between them. In addition, the Coulomb interactions are present, and their effects on the charge, orbital and magnetic order are important $[9,38,41,46,67]$. In the next section, we motivate a model recently proposed by Brink and Khomskii [36] for the electron-doped manganites and later extended by us [38] in order to take into account the effects of local Coulomb interactions present in these systems. We extend this model further in the present work, study the magnetic and orbital orders in more detail, investigate the possibility of charge-ordering and phase separation and 
discuss their consequences. In sections II and III we present our calculations and results and compare them with experimental literature. We conclude with a brief discussion on the implications of our results.

\section{II.a. Degenerate Double-Exchange Model}

Evidently the physics of the region $x>0.5$ is quite different from that in the $x<0.5$ for the manganites and one has to look at the electron-doped manganites from a different perspective. In order to pay due heed to the compelling experimental and theoretical evidence in support of the vital role of the orbitals, Brink and Khomskii [36] (hereafter referred to as BK) have proposed a model for the electron-doped manganites that incorporates the $e_{g}$ orbitals and the anisotropic hopping between them. In the undoped $\mathrm{LaMnO}_{3}$ compound each Mn ion has one electron and acts as a Jahn-Teller centre, the $e_{g}$ orbitals are split and the system is orbitally ordered. Thus for the lightly (hole-) doped system one can at the first approxmation ignore the orbital degree of freedom and apply a single band model like the conventional double exchange (DE) model to describe it. If, however, one proceeds from the opposite end and starts, for example, from the insulating $\mathrm{CaMnO}_{3}$ compound where the empty $e_{g}$ orbitals of $\mathrm{Mn}^{+4}$ ions are degenerate, then doping trivalent (La, $\mathrm{Nd}, \mathrm{Pr}$ etc.) ions into $\mathrm{CaMnO}_{3}$ results in adding electrons into the doubly degenerate $e_{g}$ manifold.

In the doped manganites $\mathrm{R}_{1-x} \mathrm{~A}_{x} \mathrm{MnO}_{3}$ there are $y=1-x$ number of electrons in the $e_{g}$ orbitals at each $\mathrm{Mn}$ site. Since each site has two $e_{g}$ orbitals, four electrons can be accommodated per site and hence the actual filling (electron density) is $\frac{y}{4}$. At $x=0.5$ (or $y=0.5)$, corresponding to the maximum filling in the electron-doped regime, every alternate Mn site has one electron on the average. This means that the highest filling in the electrondoped region is only $\frac{1}{8}$ [we restrict ourselves to the region $0.5 \leq x \leq 1.0 \quad(0.5 \geq y \geq 0)$ in the foregoing]. Due to this low electron concentration and hence very few Jahn-Teller centres the $e_{g}$ band is mostly degenerate and the Jahn-Teller effect is negligible to a leading 
approximation. The neglect of Jahn-Teller effect is also justified from the experimental evidence presented above. The usual charge and spin dynamics of the conventional DE model then operate here too, albeit with an additional degree of freedom coming from the degenerate set of $e_{g}$ orbitals. This process has been described by BK as double exchange via degenerate orbitals.

In order to capture the magnetic phases properly, the model has, in addition to the usual double exchange term, orbital degeneracy and the superexchange (SE) coupling between neighbouring $t_{2 g}$ spins. At $x=1$ ( or $y=0$ ) end the $e_{g}$ band is completely empty and the physics is governed entirely by the antiferromagnetic exchange (superexchange) between the $t_{2 g}$ spins at neighbouring sites. On doping, the band begins to fill up, the kinetic energy of electrons in the degenerate $e_{g}$ levels along with the attendant Hund's coupling between $t_{2 g}$ and $e_{g}$ spins begin to compete with the antiferromagnetic superexchange interaction leading to a rich variety of magnetic and orbital structures. The model used to describe the ground state properties of the electron-doped manganites contains the following terms

$$
H=J_{A F} \sum_{<i j>} \mathbf{S}_{\mathbf{i}} \cdot \mathbf{S}_{\mathbf{j}}-J_{H} \sum_{i} \mathbf{S}_{\mathbf{i}} \cdot \mathbf{S}_{\mathbf{i}}-\sum_{<i j>\sigma, \alpha, \beta} t_{i, j}^{\alpha \beta} c_{i, \alpha, \sigma}^{\dagger} c_{j, \beta, \sigma}
$$

The first term is the usual AF superexchange between $t_{2 g}$ spins at nearest-neighbour sites, the second term represents the Hund's exchange coupling between $t_{2 g}$ and $e_{g}$ spins at each site and the third term stands for the hopping of electrons between the two orbitals $[16,63,64]\left(\alpha, \beta\right.$ take values 1 and 2 for $\mathrm{d}_{x^{2}-y^{2}}$ and $\mathrm{d}_{3 z^{2}-r^{2}}$ orbitals, corresponding to the choice of the phase $\xi_{i}=0$ in Ref.[65]). The hopping matrix elements are determined by the symmetry of $e_{g}$ orbitals $[16,63]$. Although similar in appearance to the conventional DE model the presence of orbital degeneracy together with the very anisotropic hopping matrix elements $t_{i j}^{\alpha \beta}$ makes this model and its outcome very different from the conventional DE model of Zener $[8,25,68,69]$ with a single non-degenerate orbital.

In the manner often used in literature [8] BK treated the $t_{2 g}$ spins quasi-classically and 
the Hund's coupling was set to infinity. At each site the spins were allowed to cant in the $x z$-plane leading to the effective hopping matrix elements [8] $t_{x y}=t \cos \left(\theta_{x y} / 2\right)$ and $t_{z}=t \cos \left(\theta_{z} / 2\right)$. Here $\theta_{x y}$ is the angle between nearest neighbour $t_{2 g}$ spins in the $x y-$ plane and $\theta_{z}$ is the same in the $z$-direction. The superexchange energy per state then becomes

$$
E_{S E}=\frac{J_{A F} S_{0}^{2}}{2}\left(2 \cos \theta_{x y}+\cos \theta_{z}\right)
$$

In this level of approximation, the problem reduces to solving the $2 \times 2$ matrix equation $\left\|t_{\alpha \beta}-\epsilon \delta_{\alpha \beta}\right\|=0$ for a system of spinless fermions. The matrix elements are determined using the standard form of the overlap integrals [16]

$$
\begin{gathered}
t_{11}=-2 t_{x y}\left(\cos _{x}+\cos k_{y}\right) \\
t_{12}=t_{21}=-\frac{2}{\sqrt{3}} 2 t_{x y}\left(\cos _{x}-\cos k_{y}\right) \\
t_{22}=-\frac{2}{3} t_{x y}\left(\cos k_{x}+\cos k_{y}\right)-\frac{8}{3} t_{z} \cos k_{z} .
\end{gathered}
$$

Here $t_{11}$ is the dispersion due to the overlap between $d_{x^{2}-y^{2}}$ orbitals on neighbouring sites, $t_{12}$ between a $d_{x^{2}-y^{2}}$ and a $d_{3 z^{2}-r^{2}}$ orbital and $t_{22}$ between two $d_{3 z^{2}-r^{2}}$ orbitals. In the foregoing, the system is assumed to posses a cubic unit cell without any distortion. This is not a serious drawback, as for these systems in the doping range considered, the deviations from cubic symmetry are not large [4]. Writing $t_{x y}$ and $t_{z}$ in terms of $\theta_{x y}$ and $\theta_{z}$ in the $\mathrm{J}_{H} \rightarrow \infty$ approximation, the matrix equation is easily solved to get the energy bands $\epsilon_{ \pm}(k)$ as

$\epsilon_{ \pm}(k)=-\frac{4 t_{x y}}{3}\left(\cos k_{x}+\cos k_{y}\right)-\frac{4 t_{z}}{3} \cos k_{z} \pm\left(\left[\frac{2 t_{x y}}{3}\left(\cos k_{x}+\cos k_{y}\right)-\frac{4 t_{z}}{3} \cos _{z}\right]^{2}+\frac{4 t_{x y}^{2}}{3}\left(\cos k_{x}-\cos k_{y}\right)^{2}\right)^{\frac{1}{2}}$.

We plot these energy bands in fig. 3 along different symmetry directions in the cubic Brillouin zone (BZ) in the A-phase (fig. 3a) and also (fig. 3b) in the absence of a magnetic order (i.e., for $t_{x y}=t_{z}=t$ ) to demonstrate the nature of dispersion. In the pure (uncanted) phases the bands in A- and C- phases become purely two- and one-dimensional. However, 
we have plotted the bands for the A-phase in the presence of a small canting in fig. 3a. Note that even in the presence of canting, there is almost no dispersion in the $z$-direction $(\Gamma-Z$ and M-L directions). In the canted C-phase as well the band disperses little in the $x$ and $y$ directions and remains almost indistinguishable from the pure phase.

The total energy is then obtained for a particular filling by adding the superexchange contribution to the band energy. It is evident that the energy spectrum obtained depends on the underlying magnetic structure as well as the orbital-dependent (anisotropic) hopping matrix elements. This will lead to different anisotropic magnetic structures at different doping. The magnetic phase diagram in the (electron) doping $y-t / J_{A F}$ plane is then calculated by minimizing the total energy with respect to $\theta_{x y}$ and $\theta_{z}$. The sequence of phases follows from the nature of the DOS modulated by the anisotropic overlap of orbitals as well as the DE mechanism. At very low doping $(x \sim 1)$ BK get a stable A-type (canted) antiferromagnetic phase and on increasing the doping the system first enters the C-phase and then depending on the value of $t / J_{A F}$ directly gets into the ferromagnetic phase or reenters the A-phase before becoming ferromagnetic at large doping. The presence of ferromagnetic phase at large doping and C-type antiferromagnetic order at the intermediate electron doping range is rightly captured in their model. Such a sequence of phases is indeed seen in the experimental phase diagram in these systems. Quite remarkably the phase diagram has almost all the magnetic phases except the G-type antiferromagnetic one that is observed experimentally in these systems at low electron doping. The phase diagram of BK, unfortunately, has two major shortcomings in it. At very low electron-doping a canted A-type antiferromagnetic phase is obtained which is stable for all values of $J_{A F}$ whereas experimentally G-type antiferromagnetic phase is observed at this end. The stability of the G-phase around $x \rightarrow 1$ is quite naturally expected on physial grounds. At the $y=0(x=1)$ end there are no electrons in the $e_{g}$ band, the only interaction is the antiferromagnetic exchange between neighbouring $t_{2 g}$ spins which should lead to the three dimensional G-type antiferromagnetic order. The 
other problem is that of the limiting behaviour. When the antiferromagnetic exchange interaction is zero or very close to zero (i.e. $t / J_{A F} \rightarrow \infty$ ) the system should be completely ferromagnetic, a feature which is also missed out in their phase diagram.

It appears that the designation of the A-type ordering by BK was somewhat ambiguous and that might have led to the absence of the G-phase around $x=1$ in their phase diagram. This is particularly relevant as the typical values of canting obtained by BK in their A-phase are quite large. In their convention for different spin ordering, they chose to designate Aphase when $\theta_{x y}<\theta_{z}$. It is apparent, therefore, that by this convention, a spin ordering with both the angles $\theta_{x y}$ and $\theta_{z}$ close to $\pi$ but $\theta_{x y}<\theta_{z}$, could be designated as a canted A-phase. On the other hand, from the structure of spin arrangements, it should be more appropriately called a canted G-phase. Although G-phase with such large canting has not been seen experimentally (there is hardly any evidence of significant canting in the region close to $x=1$ ). This ambiguity is easily resolved if in addition one considers orbital ordering which, however, was not included in their treatment. We discuss this in more detail later on with reference to our calculations.

The limit of infinite Hund's coupling which BK worked with is unphysical for the manganites considered $[3,9,65,67]$. Typical values reported in the experiments $[3,4,23]$ and various model studies $[9,41,65]$ and LDA calculations $[67,70]$ do not suggest the spin spilittings of the $e_{g}$ band in various manganites to be very large. These are typically comparable to (or slightly larger than) the $e_{g}$ band-width. The scale of Coulomb correlations are most likely to be even higher $[3,9]$. The other serious consequence of using such large values of Hund's coupling is that the predictions about low energy excitations (like optical spectra, specific heat, spin fluctuation energy scales) are going to be inaccurate. BK's calculation, though, serves as a starting point for improved theories.

Based on their phase diagram BK argue that the degeneracy of orbitals and the anisotropy of hopping are crucial and the lattice (including Jahn-Teller (JT) effect) is of secondary 
importance for the physics of electron-doped manganites. This was borne out by a more refined calculation by Pai. In a more realistic treatment of the spin degrees of freedom, Pai [37] considered the limit of finite $\mathrm{J}_{H}$ in the same model and succeeded in recovering the Gand F-phases.

\section{II.b. Double exchange and correlation}

We mentioned earlier that by all estimates the Coulomb correlations in these systems are large $[24,73,70]$ and it is not obvious, therefore, that the phase diagram obtained by BK will survive once these are introduced in the model. Neither of the treatments of BK or Pai includes the interactions present in the system, namely the inter- and intra- orbital Coulomb interactions as well as the longer-range Coulomb interactions. Although for low doping the local correlations are expected to be ineffective, with increase in doping they preferentially enhance the orbital ordering [38]. This affects the F-phase and alters the relative stability of the A- and C-phases. The longer-range part of the interactions would tend to localize the carriers and lead to charge ordering. It is, therefore, necessary to include them in the Hamiltonian and look for their effects on the phase diagram. In the present work we have incorporated the onsite inter- and intra-orbital as well as the nearest neighbour Coulomb interactions in the model Hamiltonian and studied how these terms affect the nature of magnetic phase diagram, orbital ordering and other properties of electron doped

manganites. We also set out from the double exchange model with degenerate $e_{g}$ orbitals and the superexchange interaction between the neighbouring $t_{2 g}$ spins. The addition of the correlation terms makes the model very different from the ones considered by BK and Pai. Besides, the physics of charge ordering is beyond the scope of the models earlier considered.

The model Hamiltonian we consider consists of two parts, the first part is the same as the Hamiltonian in eqn. (1) we discussed in the previous section. The second part, which is the interaction part, has onsite inter- and intra- orbital interaction and the nearest neighbour 
Coulomb interaction terms in it. The total Hamiltonian is therefore

$$
H=H_{1}+H_{\text {int }}
$$

$H_{1}$ is the same as in eqn. (1) and

$$
H_{i n t}=U \sum_{i \alpha} \hat{n}_{i \alpha \uparrow} \hat{n}_{i \alpha \downarrow}+U^{\prime} \sum_{i \sigma \sigma^{\prime}} \hat{n}_{i 1 \sigma} \hat{n}_{i 2 \sigma^{\prime}}+V \sum_{<i j>} \hat{n}_{i} \hat{n}_{j} .
$$

In the above $U, U^{\prime}$ and $V$ are the intra- and inter-orbital and the nearest neighbour Coulomb interaction strengths respectively. We treat the $t_{2 g}$ spin subsystem quasi-classically as in BK (this is the usual practice in many of the treatments of the double exchange model $[8,9,68])$, but we choose to work with the more realistic limit of finite values of the Hund's coupling. In an uncanted homogeneous ground state we choose $\mathbf{S}=\mathbf{S}_{0} \exp (i \mathbf{Q} \cdot \mathbf{r})$ where the choice of $\mathbf{Q}$ determines the different spin arrangements for the core $\left(t_{2 g}\right)$ spins. For example, $\mathbf{Q}=(0,0,0)$ would be the pure ferromagnetic phase, $\mathbf{Q}=(\pi, \pi, \pi)$ gives the G-type antiferromagnetic phase, $\mathbf{Q}=(\pi, \pi, 0)$ is for $\mathrm{C}$ - type antiferromagnetic phase and finally $\mathbf{Q}=(0,0, \pi)$ reproduces A-type antiferromagnetic phase. In the infinite $\mathrm{J}_{H}$ limit, the $e_{g}$ electron spins are forced to follow the $t_{2 g}$ spins leading to the freezing of their spin degrees of freedom. At finite $\mathrm{J}_{H}$, however, the quantum nature of the transport allows for fluctuations and the $e_{g}$ spin degrees of freedom, along with anisotropic hopping across the two orbitals, play a central role. For canted magnetic structures where the angle between two nearest-neighbour $t_{2 g}$ spins is different from that of the pure phases, $\mathbf{S}_{i}$ is given by $\mathbf{S}_{i}=S_{0}\left(\sin \theta_{i}, 0, \cos \theta_{i}\right)$ with $\theta_{i}$ taking all values between 0 and $\pi$. The $t_{2 g}$ spins are allowed to cant only in the $x z$-plane (this does not cause any loss of generality in the treatments that follow). We will discuss the canted structures at length in the foregoing. We begin our discussion by considering the model without the interaction terms $U, U^{\prime}$ and $V$. The interactions and their effects will be dealt with in detail later. 


\section{II.c. The non-interacting limit}

Using the usual semi-classical approximation for the $t_{2 g}$ spins and the choice $\mathbf{S}=\mathbf{S}_{0} \exp (i \mathbf{Q} . \mathbf{r})$, the Hamiltonian (1) reduces in the momentum space to

$$
H=\sum_{\mathbf{k}, \alpha, \beta, \sigma} \epsilon_{\mathbf{k}}^{\alpha \beta} c_{\mathbf{k} \alpha \sigma}^{\dagger} c_{\mathbf{k} \beta \sigma}-J_{H} S_{0} \sum_{\mathbf{k}, \alpha} c_{\mathbf{k} \alpha \uparrow}^{\dagger} c_{\mathbf{k}+\mathbf{Q} \alpha \uparrow}+J_{H} S_{0} \sum_{\mathbf{k}, \alpha} c_{\mathbf{k} \alpha \downarrow}^{\dagger} c_{\mathbf{k}+\mathbf{Q} \alpha \downarrow}
$$

where we have followed the notation in $[16,64] . \epsilon_{\mathbf{k}}^{\alpha \beta}$ are the same as $t^{\alpha \beta}$ introduced in eqn.

We can see from the above Hamiltonian that the matrix is now an $8 \times 8$ one with two spins (up and down), two degenerate orbitals $\left(d_{x^{2}-y^{2}}\right.$ and $\left.d_{3 z^{2}-r^{2}}\right)$ with (anisotropic) hopping between them and two momentum indices $(\mathbf{k}$ and $\mathbf{k}+\mathbf{Q})$. Thus, taking a finite value of $J_{H}$ makes the problem $8 \times 8$ one at each $\mathbf{k}$-point in contrast to $2 \times 2$ spinless problem for infinite $J_{H}$ treated in BK. The superexchange part of the ground state energy per state is coming from the first term in $H_{1}$ and is the classical contribution $E_{S E}=\frac{J_{A F} S_{0}^{2}}{2}\left(2 \cos \theta_{x y}+\cos \theta_{z}\right)$.

We diagonalize the Hamiltonian in eqn. (6) at each $\mathbf{k - p o i n t}$ on a finite momentum grid. The numerical results converged by a grid size of $64 \times 64 \times 64$. The ground state energy is calculated from the eigenvalues for different magnetic structures (F, A, C and G) in their uncanted configurations. The magnetic structure with minimum ground state energy is determined for each set of parameters $\left(x, J_{H}\right.$ and $\left.J_{A F}\right)$ for the entire range of electron doping $(0.5 \leq x \leq 1)$ to obtain the magnetic phase diagram. In fig. 4 we show the ground state energies for different magnetic structures for $J_{A F} S_{0}^{2}=0.05$ and $J_{H} S_{0}=16$ around the transition points in the doping range $0.5 \leq x \leq 1.0$. The value of $J_{H} S_{0}$ is chosen somewhat large to compare the figure with that when $U^{\prime} \neq 0$ later. All energies are measured in units of t. The figure shows that there is a G-type AFM to C-type AFM phase transition occuring at $x=0.91$, C-type to A-type transition at $x=0.62$ and the A-type AFM to F (ferromagnet) transition at $x=0.57$. The procedure is repeated for different values of $\mathrm{J}_{A F}$ keeping $\mathrm{J}_{H}$ fixed and then reversing the order to generate the full phase diagram. 


\section{II.d. Magnetic phase diagram and canting}

The phase diagram in the $x-J_{H} S_{0}$ plane for a typical value of $J_{A F} S_{0}^{2}=0.05$ is shown in fig. 5. There is no general agreement on the values of the parameters involved [9]. From photoemission and optical studies [9] and LDA analysis [70] one can glean a range of typical values [67]: $0.1 \mathrm{eV}<t<0.3 \mathrm{eV}, J_{H} \simeq 1.5-2 \mathrm{eV}$ and $\mathrm{J}_{A F} \simeq 0.03 t-0.01 t$, (Maezono et al. $[41,42]$, though, quote a lesser value of $\mathrm{J}_{A F}=0.01 t$, the source of which is a possible use of antiferromagnetic transition temperatures in these systems to deduce the value of $\mathrm{J}_{A F}$ ). We observe that for low values of $\mathrm{J}_{H} S_{0}$ A-type antiferromagnetic phase is stable near $x=0.5$, then C-phase is stabilized for a wide region in the intermediate doping range and finally near $x=1$ the G-type antiferromagnetic phase has a lower energy. For higher values of $J_{H} S_{0}$ the ferromagnetic phase has the lowest energy near $x=0.5$ and the sequence of magnetic phases from $x=0.5$ to $x=1$ is $\mathrm{F} \rightarrow \mathrm{A} \rightarrow \mathrm{C} \rightarrow \mathrm{G}$. All the tranisitions appear to be continuous without any jump in the magnetic order parameters. The general trend observed here is in good accord with the experimental phase diagram of the electron-doped manganites of intermediate bandwidth such as $\mathrm{Nd}_{1-x} \mathrm{Sr}_{x} \mathrm{MnO}_{3}, \mathrm{Pr}_{1-x} \mathrm{Sr}_{x} \mathrm{MnO}_{3}[28,34,26]$. Unlike BK we find the G-type antiferromagnetic phase to be stable near $x=1$. This is also in agreemnet with Pai [37].

At the $x=1$ end the degenerate $e_{g}$ orbitals are completely empty. The superexchange interaction is isotropic and leads to a three dimensional G-type antiferromagnetic phase. At low electron-doping the superexchange still wins over the kinetic energy gain of the electrons via the development of a ferromagnetic component of spins in the DE mechanism. Thus the G-phase is stable up to a finite electron doping. The value of $x$ where G-phase becomes unstable depends weakly on $\mathrm{J}_{H}$ in the experimentally relevant range of $\mathrm{J}_{H}$. On further increasing the electron doping, the kinetic energy starts dominating over the superexchange contribution leading to increased spin alignment. This happens because the kinetic energy 
is an increasing function of doping and for small doping it is proportional to the (electron) filling whereas the superexchange energy is nearly independent of $x$ [77]. A three-dimensional antiferromagnetic spin alignment such as G-phase does not allow the electrons to delocalize for the typical values of $\mathrm{J}_{H}$. To gain the kinetic energy the system tries to polarize the spins along one, two and finally in all three directions successively in the sequence C-, Aand F-phases. The gain in the kinetic energy due to such alignments more than offsets the loss in the superexchange energy above a particular filling. Thus C-type antiferromagnetic phase with ferromagnetically aligned spins along the $z$-direction appears first as we increase the electron doping. Then the A-type AFM phase with a two-dimensional spin alignment appears and finally the ferromagnetic phase with complete alignment of spins is observed.

The stability of A- and C-phases are further enhanced by the ordering of orbitals in these phases. As we show below, the A-phase has an orbital ordering of $d_{x^{2}-y^{2}}$ type and the C-phase has an orbital-ordering of $d_{3 z^{2}-r^{2}}$ type. The planar $d_{x^{2}-y^{2}}$ orbital-order in the $x y$-plane in the A-phase and rod-like $d_{3 z^{2}-r^{2}}$ orbital-order in the $z$-direction in the C-phase facilitate the hopping of electrons (along the plane for A-phase and across it for the Cphase) with a gain in the kinetic energy which stabilizes these phases in the respective doping ranges. Hence, it is primarily the orbital-order that regulates the DE mechanism and leads to the C- and A-type magnetic orders. Such a scenario has been borne out in several experiments $[27,34,49]$ where evidence for orbital ordering is seen at a much higher temperature than the spin ordering. However, in the G-and F-phases no significant orbital ordering has been observed. Thus the interplay of spin alignment along chains or planes and the corresponding orbital order leads to the transformation from the one-dimensional to the two-dimensional and finally to the three dimensional magnetic structure with increased doping. The competition between effective kinetic energy (determined by $\mathrm{J}_{H}$, band-filling and orbital-ordering) and superexchange leads to the transitions $\mathrm{G} \rightarrow \mathrm{C} \rightarrow \mathrm{A} \rightarrow \mathrm{F}$ (with the number of antiferromagnetic bonds 6, 4, 2 and 0 per site respectively) as the doping is varied 
for a given set of values of $J_{H}$ and $J_{A F}$. The dimensionality of the magnetic and orbitalorder in the A- and C-phases described above is reflected in the density of state (DOS) in these phases. In the A-type AFM phase the dispersion of bands is two-dimensional with a peak near the centre of the band and small but nonzero DOS at the band edges when the hopping $t_{12}$ between $d_{x^{2}-y^{2}}$ and $d_{3 z^{2}-r^{2}}$ orbitals is zero. For a finite $t_{12}$ the DOS is still two-dimensional, but the peak at the centre splits. In the canted C-phase the DOS is quasione-dimensional (for $t_{x y}=0$ it becomes purely one-dimensional) with peaks towards the band edges. In the pure C-phase the band disperses only in the $z$-direction and the DOS is one-dimensional.

Experimentally $[27,34]$ it is observed that there is little canting in A- and C-phases in most of these systems. This was also emphasized by Maezono et. al. [87] from their theoretical analysis. There are some experimental observations [71] on $\mathrm{Sm}_{1-x} \mathrm{Ca}_{x} \mathrm{MnO}_{3}$ which suggest that the G-phase, for low doping, has small canting. Canting of the core spins is included in our calculation by writing $\mathbf{S}_{\mathbf{i}}=\mathbf{S}_{0}\left(\sin \theta_{i}, 0, \cos \theta_{i}\right)$ in the Hamiltonian with $\theta_{i}$ taking values between 0 and $\pi$. Such a canted spin configuration connects two different spin species (up and down) at the same site in contrast with the pure (uncanted) phase. With this choice of $\mathbf{S}_{\mathbf{i}}$, the Hund's coupling term between $\mathrm{t}_{2 g}$ and $\mathrm{e}_{g}$ spins in the Hamiltonian becomes

$$
H_{h u n d}=-J_{H} S_{0} \sum_{i, \alpha} \cos \theta_{i}\left(c_{i \alpha \uparrow}^{\dagger} c_{i \alpha \uparrow}-c_{i \alpha \downarrow}^{\dagger} c_{i \alpha \downarrow}\right)-J_{H} S_{0} \sum_{i, \alpha} \sin \theta_{i}\left(c_{i \alpha \uparrow}^{\dagger} c_{i \alpha \downarrow}+c_{i \alpha \downarrow}^{\dagger} c_{i \alpha \uparrow}\right) .
$$

In case of canted magnetic structures the different magnetic phases need to be defined at the outset. The convention (used by BK as well) to define the magnetic phases are: The phase is A-type when $\theta_{x y}<\theta_{z}$ as the spins in the $x y$-plane have more ferromagnetic component than the spins across the planes. Similarly, in the C-phase $\theta_{x y}$ is taken to be greater than $\theta_{z}$. In the canted $\mathrm{G}$ and $\mathrm{F}$ phases both $\theta_{x y}$ and $\theta_{z}$ are close to $180^{\circ}$ and $0^{0}$ respectively, although, it is then obvious that the canted G-phase and A-phase are synonymous in a certain region 
[72]. However, orbital order can be used to delineate the two phases.

The ground state energy for different $\theta_{x y}$ and $\theta_{z}$ is obtained in exactly the same manner as described above (with $\mathrm{U}$ and $\mathrm{U}^{\prime}$ set to zero). The qualitative nature of the phase diagram is very similar to the uncanted phase diagram except for little shifts in the phase boundaries (the shifts are small unless $\mathrm{J}_{H}$ is large). We show in fig. 6a the angle of canting (for both $\theta_{z}$ and $\theta_{x y}$ ) as a function of $\mathrm{J}_{H}$ deep inside the G-phase at $x=0.98$ (the angles in fig. 6 represent deviation from $180^{\circ}$ ) for different values of $J_{A F} S_{0}^{2}$.

There is hardly any canting in the $z$-direction while in the $x y$-plane there is no significant canting for low $J_{H}$ and it is about $10^{\circ}$ only for large $J_{H}$. So, for realistic values of $J_{H}$, there is no observable canting. The absence of canting in $\theta_{z}$ is seen for all the different values of $J_{A F} S_{0}^{2}$. An increase in $J_{A F}$ reduces canting of $\theta_{x y}$ (fig. 6b) and stabilizes the pure G-phase as expected. Changing $y$ and moving closer to the boundary with the Cphase, canting in $\theta_{x y}$ is seen to increases quite slowly. However, very close to the G-C boundary, $\theta_{x y}$ reverts back towards $\pi$ while $\theta_{z}$ begins to deviate from $\pi$. In the G-phase, small canting has been reported in certain systems as discussed earlier. For very low electrondoping the superexchange interaction wins over double exchange and the phase is G-type antiferromagnetic. On doping, the electrons would try to delocalize. Since it is energetically costly ( $J_{H}$ being the largest scale) for electrons to move in a completely antiferromagnetic configuration it is expected that the system will try to gain kinetic energy via the canting of the core spins. The canting angle will be anisotropic, i.e., $\theta_{z}$ will be different from $\theta_{x y}$ due to the anisotropy of $t_{i j}^{\alpha \beta}$. We should also note the fact that canting in the plane leads to higher gain in kinetic energy than what is gained by canting in the $z$-direction. This does not, however, mean that the phase that abuts G-phase as $x$ decreases would be the planar (A-type) magnetic phase - the values of the two angles are delicate functions of doping, the dimensionality of the DOS as well as the anisotropy of the hopping integral. The phase that appears after G-phase with increased doping is the C-phase. In the $\mathrm{J}_{H}$ inifinite limit electron 
hopping to neighbouring sites with antiparallel core spins is not allowed because the effective hopping parameter in this case is proportional to $\operatorname{tcos}(\theta / 2)$ where $\theta$ is the angle between the spins at neighbouring sites and antiparallel arrangement of spins reduces it to zero. Hence the only way the electrons can take advantage of the kinetic energy gain due to increased doping is by canting the core spins as much as possible (at the cost of superexchange energy, which is, however, small). This will give rise to a ferromagnetic moment so that the electrons can hop from site to site. BK had projected out the "wrong" spin sector of the Hilbert space in their effective theory with infinite Hund's coupling. This is why they observed large canting of spins in the A- and C-phases. However, at finite $\mathrm{J}_{H}$ this picture is changed altogether. The wrong spins are no longer as "costly" and a finite value of $\mathrm{J}_{H}$ allows an electron in the wrong spin state with an energy cost proportional to $J_{H}$. Hence the canting angle reduces drastically as compared to the $J_{H} \rightarrow \infty$ limit. In fact, for experimentally realistic values of $J_{H}$ the canting is almost negligible as can be inferred from fig. 6 . It is to be noted that a small canting in the $x y$-plane in the G-phase gives rise to a net ferromagnetic correlation in the plane with a value higher than that across the layers (which is zero if there is no canting in the $z$-direction). Hence one could think of it as a canted A-phase following the convention of BK. However, the orbital order, which is present in the A-phase, but absent in the G-phase, can be used to distinguish these two phases. Moreover, the kinetic energy gain, which, for small doping, is proportional to the doping, is not effective in overcoming the SE energy unless $x$ deviates from 1 reasonably. Hence one gets a canted G-phase with very small canting angles in the region close to $x=1$, resembling the end-member pure G-phase. Since the kinetic energy gain is quite small due to the small values of the canting angle, this phase does not have any preferential orbital arrangement of the $d_{x^{2}-y^{2}}$ or $d_{3 z^{2}-r^{2}}$ type as in the C- and A-phases. Thus the stability of the G-phase is primarily due to the dominance of SE energy in the region close to $x=1$. This also means that the doping region over which the G-phase stabilizes will grow with increase in $\mathrm{J}_{A F}$. In particular, for $\mathrm{J}_{A F} \rightarrow 0$ the system 
should exhibit ferromagnetism for any doping. However, BK find that the phase boundary between the canted G-phase and the C-phase does not change significantly as $\mathrm{J}_{A F}$ is varied. In contrast, the phase diagram we obtained gives a ferromagnetic state for $\mathrm{J}_{A F} \rightarrow 0$ for the entire doping regime and the stability region of the G-phase grows with increase in $J_{A F}$ as expected. Our results agree in general with the results of Maezono et. al.[87] though the A-phase near $x=0.5$ is missing in their work. In a related work, Sheng and Ting [74] considered the problem from the strong correlation point of view in contrast to the band limit that we have adopted. They obtained an effective model with coupled spin and orbital degrees of freedom in the strong-interaction limit and use Monte Carlo method to study this model. The C-phase, however, could not be obtained from their model anywhere in the range $x \geq 0.5$.

\section{III.a. The interacting case: magnetic phases}

We treat the three interaction terms in the Hamiltonian (5) in the mean-field theory. It has been pointed out by Hotta et al. [65], that the mean-field theory for the interacting double-exchange model even in low dimension gives very good agreement with exact diagonalization on small systems. Comparison of mean-field phase diagram with exact diagonalization on small systems by Misra et al. [67] is also quite encouraging. Let us first look into the inter-orbital Coulomb interaction term $U^{\prime} \sum_{i \sigma \sigma^{\prime}} \hat{n}_{i 1 \sigma} \hat{n}_{i 2 \sigma^{\prime}}$ and set $U=V=0$ in $H_{\text {int }}$. In the mean-field theory, one neglects fluctuations and writes $\hat{n}_{i 1 \sigma} \hat{n}_{i 2 \sigma^{\prime}}=<\hat{n}_{1 \sigma}>\hat{n}_{i 2 \sigma^{\prime}}+<$ $\hat{n}_{2 \sigma^{\prime}}>\hat{n}_{i 1 \sigma}-<\hat{n}_{1 \sigma}><\hat{n}_{2 \sigma^{\prime}}>$.

The homogeneous averages $\left\langle\hat{n}_{1 \uparrow}\right\rangle,\left\langle\hat{n}_{1 \downarrow}\right\rangle,\left\langle\hat{n}_{2 \uparrow}\right\rangle,\left\langle\hat{n}_{1 \downarrow}\right\rangle$ were calculated iteratively through successive diagonalization of the Hamiltonian. Each of the average quantities and the filling were calculated from the resultant eigenvectors for a chosen chemical potential and fed back to the Hamiltonian for next iteration. All the averages and filling were thus allowed to reach self-consistent solutions. Self-consistency is achieved when all averages and 
the ground state energy converge to within $0.01 \%$ or less (depending on the difference in energy with the competing ground state). In this way the ground state energies are calculated at each filling for all four magnetic phases (F, A, C and G) and the minimum energy phase was determined to obtain the complete magnetic phase diagram in the entire electron-doping regime by varying both $\mathrm{J}_{H}$ and $\mathrm{J}_{A F}$.

We show in fig. 7 the ground state energies of different magnetic phases around the transition points with $J_{A F} S_{0}^{2}=0.05, J_{H} S_{0}=16$ and $U^{\prime}=8$. We see that the G-C phase transition occurs at $x=0.91$ as before, C-A phase transition at $x=0.57$ and the A-F phase transition at $x=0.51$. Comparing this with fig. 4 we note the shift of position of the transitions. The G-phase remains uaffected, the C-phase widens and F-phase shrinks for $U^{\prime}>0$. The phase diagram in the $x-J_{H}$ plane for $J_{A F} S_{0}^{2}=0.05$ and $U^{\prime}=8$ is shown in fig. 8. The panel from 8(a)-(c) show the progession of the phase diagram as $U^{\prime}$ increases. The $U^{\prime}=0$ phase diagram is shown in $8($ a) by dashed lines for comparison.

It is observed that on increasing $U^{\prime}$ the ferromagnetic phase starts shrinking fast, the C-phase gains somewhat while the G-phase remains almost unaltered for the entire range of values of $\left.J_{H} S_{0}\right)$ studied. The trends observed here are in good agreement with the experimental observations of Kajimoto et al. [28, 34] and Akimoto et al. [27] (see figs. 1 and 2). The enhanced correlation effectively reduces the phase space for the electrons. The observation $[27,28]$ that on decreasing the band-width, the ferromagnetic phase shrinks and finally gets pushed below $x=0.5$ with A-phase becoming stable at $x=0.5$ is borne out in fig. 8 . The stabilities of A- and C-phases are primarily derived from the enhanced orbital-ordering in the A- and C-phases driven by the inter-orbital repulsion and the low dimensional nature of the DOS. In the presence of $U^{\prime}$, the one-dimensional order leading to the AF instability in the C-phase seems to grow faster. Close to the $x=1$ end the electron density is very low, there are almost no sites with both the orbitals occupied and $U^{\prime}$ is therefore ineffective. The G-phase remains almost unaffected as in fig. 1. Similarly the canting of the spin away from 
$\pi$ observed in the G-phase remains the same as in fig. 6. At the other end, however, the electron-density is higher and the F-phase has preferential occupation of one species of spin at both the orbitals. Hence this phase is affected drastically by the inter-orbital repulsion.

We also compare the phase diagrams with and without $U^{\prime}$ in the $x-J_{A F}$ plane for $J_{H} S_{0}=10$. The corresponding phase diagram is shown in fig. 9. Trends observed in fig. 5 and fig. 8 are also seen in this case. The topology of the phase diagram has not changed, though the A-phase and F-phase shrink in presence of $U^{\prime}$ while the C-phase has grown.

It is known [65] that at the level of mean-field theory the intra-orbital repulsion $U$ between opposite spins mimics the effect of $J_{H}$. As we are working with quite low densities (actual filling $\leq 0.125)$, and the relevant $J_{H}$ values are moderate to large, there is hardly any site with both spin species present. Therefore, we find almost no observable effect of $U$ on the phase diagram (except for very low $J_{H}$ where again the changes are small) and keep its value zero in the phase diagrams shown.

\section{III.b. Magnetic ordering and disorder}

The doped manganites $\mathrm{R}_{1-x} \mathrm{~A}_{x} \mathrm{MnO}_{3}$ are intrinsically disordered owing to the substitution of trivalent ions by divalent ones. Although the dopant ions do not enter the active network of $\mathrm{MnO}_{6}$ octahedra that are considered central to the transport properties and magnetic ordering, their effects cannot be ignored. In this kind of substitution not only are the charges on the dopant ions different from the trivalent rare-earth ions they replace, the ionic sizes of the rare-earths vary considerably (e.g., La, Nd, Pr all have different ionic sizes). Hence there is a mismatch of ionic sizes between these and the divalent ion (like Sr, Ca etc.) that

replaces them. Such mismatch would quite naturally bring about large lattice distortions locally.

However, the effect of disorder has been completely ignored in the treatments discussed so far. BK and Pai [37] argue that to a first approximation, the disorder does not seem to 
play a major role in the magnetic phase diagram in this region of doping. This is possibly due to the non-magnetic nature of the disorder - the rare earth ions are not found to have any observable moment except for Pr and it has been shown that Pr-Mn coupling does not have a detectable effect [66] in the magnetic structure. The lattice effects are, in any event, pronounced only close to $x=1$.

Since the Mn ions are central to the mechanism of magnetic and orbital order in the manganites, substitution at this site would be quite revealing. In the last few years quite a few experimental investigations $[78,79]$ have been carried out by substitution of Mn by Fe, Ga, Al. These have similar ionic sizes and valences as $\mathrm{Mn}$ and therefore cause very little distortion in the lattice [78] (though Al-substitution has stability problem beyond about 10\%). For example, the substitution of $\mathrm{Mn}^{+3}$ by $\mathrm{Fe}^{+3}$ (which has identical ionic size as $\mathrm{Mn}^{+3}$ [82]) in $\mathrm{La}_{1-x} \mathrm{Ca}_{x} \mathrm{Mn}_{1-y} \mathrm{Fe}_{y} \mathrm{O}_{3}$ in the AFM region at $x=0.53$ shows that the resistivity increases and magnetoresistance disappears by about $y=0.13$. Although the $\mathrm{Fe}^{+3}$ has a higher moment than the $\mathrm{Mn}^{+3}$ that it replaces, one observes a steady suppression of the magnetic moment and ferromagnetism with Fe doping [78]. Whether there is any accompanying changes in the underlying magnetic ordering is not clear. Also the systematics across several manganites with different band-widths are also not available yet.

There are two things that happen when Fe is doped in place of Mn: i) In the octahedral crystal field the $\mathrm{Fe}^{+3}$ (high-spin $\mathrm{d}^{5}$ configuration) sites have all their $\mathrm{e}_{g \uparrow}$ orbitals filled up and hence forbid the motion of electrons from $\mathrm{Mn}^{+3}$ into $\mathrm{Fe}^{+3}$ sites thereby preventing DE mechanism to operate and ii) the presence of an $\mathrm{Fe}^{+3}$ instead of $\mathrm{Mn}^{+3}$ in any site alters the superexchange interaction between this and the neighbouring sites. It is possible to account for these effects in a qualitative manner following Alonso et al [94].

The fraction of $\mathrm{Mn}^{+4}$ sites (that is the depletion in the number of electrons in the system) is increased by $(1-y)^{-1}$ when $y \neq 0$ as compared to $y=0$. For the range of $y$ Ahn et al.[78] work with $(y \sim 0.10)$, this is only about $10 \%$. So the effective depletion of electrons and 
effect (i) can be neglected to a first approximation deep inside any given phase. Similar situation obtains when $\mathrm{Al}^{+3}$ or $\mathrm{Ga}^{+3}$ (having filled d-band) are doped.

The change in the SE interaction is approximated by estimating the change in the effective antiferromagnetic interaction between neighbouring core-spins owing to the changed values of them in the coupling of Mn-Mn, Mn-Fe and Fe-Fe. The new (effective) $\mathrm{J}_{A F}$ is given by

$$
J_{A F}^{e f f}=J_{A F}\left[(1-y)^{2}+\frac{5}{3} 2 y(1-y)+\frac{25}{9} y^{2}\right]
$$

The prefactors $(25 / 9,5 / 3$ and 1$)$ come from the new spin values involved and the factors $(1-y)^{2}$ etc. are for counting the probability of sites with Mn-Mn, Mn-Fe and Fe-Fe bonds respectively. Then, at $y=0.12$ for example, the effective $J_{A F}$ is about 0.06 if the initial value of $\mathrm{J}_{A F}$ is 0.05 . This will enhance the $A F$ tendencies (and can even take the system from F- to A-type AFM phase as in fig. 9 for $x$ close to 0.5) and increase the resistivity as observed by Ahn et al.

Although a smaller effect, the depletion of the effective number of electrons taking part in the DE mechanism will reduce the conductivity and move the effective doping $x$ towards right in the phase diagram and increase AF correlations and resistivity further. There is also the possibility that due to these combined effects, the magnetic ground state may get altered, a possibility only further experiments will reveal.

There is another source of scattering coming from the localized $t_{2 g}$ spins at each Mn site. The itinerant $e_{g}$ electrons, in a mean-field sense, can be thought of as moving in a magnetic "field" of the localized spins. It has been shown [80] that such a random field can indeed localize part of the electronic states, particularly in the low-dimensional bands (as obtain in C- and A-phases). Replacing $\mathrm{Mn}^{+3}$ by $\mathrm{Fe}^{+3}$ with a different moment (5/2 as opposed to 2) provides random changes in this field and additional channel for scattering. The observation [83] of a spin-glass type phase at low temperature in the Cr-doped $\mathrm{La}_{0.46} \mathrm{Sr}_{0.54} \mathrm{Mn}_{1-y} \mathrm{Cr}_{y} \mathrm{O}_{3}$ $(0<y<0.08)$ is a possible indication of how the competing interactions between the 
coexisting FM phase in the metallic A-type AFM matrix is affected by scattering off the random magnetic Cr-impurity and the resultant localization of mobile charge carriers.

\section{III.c. Orbital ordering}

In the non-interacting case we observed orbital order in both A-phase $\left(d_{x^{2}-y^{2}}\right.$ type $)$ as well as in the C-phase (of $d_{3 z^{2}-r^{2}}$ type). Such orbital order is also borne out in experiments as discussed above. In the interacting situation, we calculate the orbital occupancies (or the orbital density as is customarily called by other workers) from the eigenvectors corresponding to the converged ground state solutions for both $d_{x^{2}-y^{2}}$ and $d_{3 z^{2}-r^{2}}$ orbitals in A- and Cphases in their respective regions of stability and show the results in fig. 10. As one can see, in the A-phase the $d_{x^{2}-y^{2}}$ orbital has a higher occupancy whereas in the $\mathrm{C}$ phase it is reversed. We check that the sum of the occupancies of two orbitals is equal to the actual filling in both the phases. The three-dimensional magnetically ordered F- and G-phases, however, show no orbital ordering, the occupancies in both the orbitals are the same.

The presence of inter-orbital Coulomb interaction $U^{\prime}$ enhances the orbital ordering in both A- and C-phases as shown in fig. 10. for three different $U^{\prime}$. Note that at lower electron densities, i.e., as $x$ increases, the effect of $U^{\prime}$ on the orbital occupancies becomes less pronounced and the curves for different $U^{\prime}$ merge as expected. We also show the orbital occupancies as a function of $U^{\prime}$ in fig. 11a,b in the regions where A- and C-phases are stable and the effect of $U^{\prime}$ is noticeable in both the A- and C-phases. The orbital densities in C-phase (fig. 11b) attain their saturation values by $U^{\prime} \simeq 8$. Since we are interested in the region $x \geq 0.5$, we have not plotted the orbital densities in A-phase (fig. 11a) beyond $U^{\prime}=8$ - above this value A-phase shifts below $x=0.5$ at $J_{H} S_{0}=5$. In the large $U^{\prime}$ limit and in the absence of $J_{A F}$ and $V$, the Hamiltonian can be mapped onto a pseudospin Hubbard model $[24,90]$ with off-diagonal hopping (that breaks the $S U(2)$, while still retaining the global $U(1)$ symmetry). Such a model overestimates the orbital order [90] and the orbital-paramagnetic 
state is almost never obtained.

The orbital order obtained in the A- and C-phases leads to anisotropic band structures in these phases and this feature becomes sharper as $J_{H}$ increases. In particular, the C-phase has a quasi one-dimensional density of states. Ideally, this phase should be conducting in the $z$-direction along the ferromagnetic chains while insulating in the plane. However, experimentally one finds this phase to be non-metallic. The nearly one-dimensional nature of transport makes it very sensitive to disorder, possibly localizing the states. In the A-phase the nature of the occupied orbitals impedes electron motion along the $z$-direction, giving rise to a large anisotropy between the in-plane and out-of-plane resistivities. Therefore the A-phase with its planar ferromagnetic alignment (and quasi 2D DOS) is not as sensitive to disorder and this rationalizes the (in-plane) metallic behaviour in the A-phase seen in several experiments $[27,28,26]$ while the $\mathrm{C}$-phase remains non-metallic.

\section{III.d. Charge ordering}

The nearest-neighbour Coulomb interaction term $V \sum_{<i j>} \hat{n}_{i} \hat{n}_{j}$ is also treated in the mean-field theory with $\left\langle\hat{n}_{i}\right\rangle=n+C_{0} \exp \left(i \mathbf{Q} \cdot \mathbf{r}_{i}\right)$ where $C_{0}$ is the charge-order parameter and $n$ is the average number of electrons per site and here $\mathbf{Q}=(\pi, \pi, \pi)$. We calculate the charge-order parameter $C_{0}$ self-consistently. A non-zero $C_{0}$ implies the presence of charge ordering. Keeping $U^{\prime}=0$, the major change observed in the phase diagram now is the

absence of the A-phase and the presence of charge ordering for values of $V>0.29$. The typical values of $V$ are between 0.2 to $0.5[3,9]$ in units of $t$. Below $V=0.29$, we do not observe any charge-ordering and A-phase reappears. The phase diagrams in the $x-J_{H} S_{0}$ plane are shown in fig. $12(\mathrm{a})$-(c) with $V=0.4, V=0.5$ and $V=0.6$ at $U^{\prime}=0$. Note that there are only three phases now. A coexisting charge-ordered ferromagnetic phase, the orbitally ordered C-phase and the G-phase. The topology does not change appreciably when $U^{\prime}$ is finite. The resultant phase diagram is shown in fig. 13. The pattern reflects what is 
seen in figs. 8 (a)-(c). The F-CO phase reduces while the C-phase grows slightly with $U^{\prime}$.

In figs. 12 and 13 a wide region of ferromagnetic charge-ordered (CO) phase is observed near $x=0.5$. This observation is in agreement with the recent experiments $[27,28]$ where the charge-ordered phase at $x=0.5$ is claimed to be ferromagnetic in nature (or possibly residing at the boundary of the F- and A-phases and straddling both). Although the coexisting F$\mathrm{CO}$ region that we get is considerably wider than the region observed experimentally. We do not find any self-consistent solution with both A-phase and charge ordering in these phase diagrams for any $V$. It is possible that the charge ordering instability is too strong close to commensurate $(x=0.5)$ filling. The A-phase, being also close to $x=0.5$ and deriving its stability from a low-dimensional density of states, gets affected by the charge order instability. Both C- and G-phases are seen to have no charge ordering in them. The CE-phase at $x=0.5$ seen in the low band-width systems has a charge stacking along the $z$-direction. Such a stacking is not favoured by the near-neighbour Coulomb term and the $\mathrm{CO}$ state obtained here has staggered charge ordering in all directions.

Our observation of the ferromagnetic charge ordered (F-CO) ground state agrees qualitatively with the mean-field calculation of Jackeli et. al. [75]. They considered a Hamiltonian that has orbital degeneracy, Hund's exchange, super-exchange and the near-neighbour Coulomb term and studied the ground state phase diagram as $V$ and $J_{A F}$ change. There is no local Coulomb term in their model. They restrict their calculations to $x=0.5$ and $J_{H} \rightarrow \infty$ limit (using effective hopping integrals) only. They obtained charge ordered F, A, $\mathrm{C}$ and G-phases in the $J_{A F} S_{0}^{2}-V$ plane when the degeneracy of the $e_{g}$ orbitals is neglected. In the degenerate model, the $\mathrm{F}-\mathrm{CO}$ phase appears only at a critical value of $V \approx 0.7$. There is no A-phase till $J_{A F} S_{0}^{2}$ reaches 0.1. All the transitions from F-CO phase into AFM states are first order.

We do indeed find a critical value of $V$ for the F-CO phase to appear. The critical value of $V$ for $J_{H} S_{0}=8$ and $J_{A F} S_{0}^{2}=0.05$ at $x=0.5$ is about 0.3 , well below the value at $J_{H} \rightarrow \infty$ 
limit. The larger value of critical $V$ is an artefact of the $J_{H} \rightarrow \infty$ limit. The tendency to large canting away from pure AF spin structures is markedly reduced in the finite $J_{H}$ limit as we discussed above. The infinite $J_{H}$ limit is, therefore, expected to overestimate the critical value of near-neighbour repulsion responsible for $\mathrm{CO}$ instability in this model as canting and eventual ferromagnetic instability with an uniform charge distribution is too strong in that limit. This critical value is nearly independent of $x$ inside the region of stability of the F-CO phase for the parameter values we considered. This is an indication of a possible phase separation (with first order transition) with part of the system pinned at the commensurate density. The $\mathrm{CO}$ order parameter $C_{0}$ has a discontinuous jump at the transition from the C-phase into the F-CO phase as shown in fig. 14, which is a signature of a first order transition between two states having different magnetic symmetry. A similar first order jump has been seen at in previous work $[67,75]$ as well and borne out in several experiments described above. The transition as a function of $V$ from pure $\mathrm{F}$ to $\mathrm{F}-\mathrm{CO}$ phase appears to be continuous (fig. 15).

\section{Discussion}

A summary of the trends observed as a function of near-neighbour interaction $U^{\prime}$ across the entire range of electron-doping is presented in fig. 16. A comparison with fig. 1 reveals the similarity between them if one interprets the increase in $U^{\prime}$ as an effective reduction in the mobility of electrons and suppression of DE mechanism. The rapid reduction in the stabilty of F- and A-phases at large $U^{\prime}$ and an almost unchanged G-phase are indeed observed in fig. 1. The C-phase is stable over a wider region of phase diagram in fig. 16 than what is experimentally observed.

There are several appealing features of the model and the limits that we have studied in the present investigation. We have been able to show that the phase diagram and orbital ordering resemble the experimentally observed ones for the electron-doped regime to a large 
degree. By putting in correlations the orbital orders are enhanced and it was possible to obtain regions of charge-ordering close to $x=0.5$. However, there are several interesting questions that need to be addressed. The neglect of Jahn-Teller effects may well describe the electron-doped manganites in the moderate to large band-width systems and also works for low band-width systems at low electron-doping. But the presence of CE-type ordering at $x=0.5$ in the entire class of low band-width materials remind us that the effects are relevant close to this doping. A more complete theory should account for the Jahn-Teller distorted $\mathrm{Mn}^{+3}$ sites and evolve from the low band-width to the large band-width description successfully. Such a theory, however, is lacking at present. In both $\mathrm{Nd}_{1-x} \mathrm{Sr}_{x} \mathrm{MnO}_{3}$ and $\operatorname{Pr}_{1-x} \mathrm{Sr}_{x} \mathrm{MnO}_{3}$ it has been seen $[26,32,28]$ that at finite temperature $(T \simeq 150 \mathrm{~K})$ there is a first order transition at $x=0.5$ from a ferromagnetic metal to an antiferromagnetic A-phase which is insulating but has quite low resistivity (immediately away from $x=0.5$ it becomes A-type metal). Kajimoto et al. [28] have also reported a stripe-like ordering in the $\mathrm{Pr}_{1-x} \mathrm{Sr}_{x} \mathrm{MnO}_{3}$ system coinciding with this weakly first order transition. There is also the possible phase separation into competing orders in this region. The model described here does reproduce a first order transition from an F-CO state to a C-type AFM state with concomitant phase separation, albeit with a large region of stability for the CO state. In real systems, with longer range Coulomb interactions present, the phase seapration is likely to appear as domains of one phase dispersed in another. Whether this indeed is the mechanism of the inhomogeneous phases observed or they are intrinsic to the systems $[51,55,59,96]$ is an open question. Transport properties in this region are going to be intriguing with possible percolative growth of FM clusters in an applied magnetic field as an alternate route to negative magnetoresistance as opposed to the DE mechanism.

Extending the model we considered with the possible inclusion of lattice degrees of freedom and from a finite temperature calculation, it should be possible to look into stripe formations and anisotropic charge orders. It has been suggested [93] recently from a finite 
temperature mean-field calculation with a degenerate, non-interacting DE model at infinite $J_{H}$ limit that without the Jahn-Teller physics brought in, the CE-phase at $x=0.5$ in the low band-width system is not accessible. Though the possibility is wide open [45] in the presence of Coulomb interactions like $U^{\prime}$ and $V$.

There is a major class of layered manganites for which the electron-doped side is still unexplored in detail. The bi-layer systems like $\mathrm{La}_{2+2 x} \mathrm{Sr}_{1-2 x} \mathrm{Mn}_{2} \mathrm{O}_{7}$ have shown [95] similar anisotropic magnetic structures as in 3D manganites. Preliminary results from a mean-field analysis [91] show interesting promise. It is difficult though to account for the large region of C-type ordering seen in experiments in such layered systems at doping $(x)$ ranges as high as $0.75-0.90$. In the layered systems the DE mechanism is expected to favour either a planar A-type or a G-type state, depending on the carrier concentration, over the 1D C-like ordering. The present model may need additional inputs in order to understand the layered manganites.

We have not looked into the excitation spectrum of the manganites so far. The effects of fluctuation coming from both spin and orbital degree and their coupling may lead to complicated excitations $[24,33,76]$. They will affect the thermodynamics quite strongly. The controlled incorporation of disorder, particularly without affecting the lattice [78, 79], has opened up a host of possibilities. The observation of non-metallic behaviour in an inhomogeneous mixture of two metallic phases [81] is an indication of the complex nature of coupling across the boundary of such domains. The spin-glass like phase reported close to the border of hole- and electron-doped region [83] in $\mathrm{La}_{0.46} \mathrm{Sr}_{0.54} \mathrm{Mn}_{1-y} \mathrm{Cr}_{y} \mathrm{O}_{3}$ is another manifestation of the complicated coupling of the impurity with spin and charge degrees of freedom. More results of such impurity doping in the electron-doped manganites are expected in the near future. We have extended the model we used to incorporate some of these effects [92] and it would be quite instructive to investigate the nature of coupling between the impurity and the magnetic and orbital degrees of freedom. 


\section{Acknowledgement}

The research of AT has been funded by a grant from the department of scince and technology, govt. of India. We acknowldge several useful discussions with S. D. Mahanti and G. V. Pai. Discussions with S. K. Ghatak and Rahul Pandit are also acknowledged. 


\section{References}

[1] Physics of Manganites, edited by T. A. Kaplan and S. D. Mahanty (Kluwer Academic, New York, 1999).

[2] Y. Tokura, Colossal Magnetoresistive Oxides, Gordon and Breach (2000).

[3] J. M. D. Coey, M. Viret and S. von Molnar, Adv. in Physics 48, 167 (1999).

[4] J. M. D. Coey, Phil. Trans. Roy. Soc. Lond. A 356, 1519 (1998).

[5] K. Chahara, T. Ohono, M. Kasai, Y. Kanke and Y. Kozomo, Appl. Phys. Lett. 62, $780(1993)$.

[6] R. von Helmholtz, J. Wecker, B. Holzapfel, I. Schultz and K. Samwer, Phys. Rev. Lett 71, 2331 (1993).

[7] P. Schiffer, A. P. Ramirez, W. Bao and S. -W. Cheong, Phys. Rev. Lett 75, 3336 (1995).

[8] C. Zener, Phys. Rev. 82, 403 (1951); P. G. de Gennes, ibid 118, 141 (1960); P. W. Anderson and H. Hasegawa, ibid 100, 675 (1955).

[9] E. Dagotto, T. Hotta and A. Moreo, Phys. Reports 344, 1 (2001),

[10] A. J. Millis, P. B. Littlewood and B. I. Shraiman, Phys. Rev. Lett 74, 5144 (1995); A. J. Millis, B. I. Shraiman, and R. Mueller, Phys. Rev. Lett. 77, 175 (1996); A. J. Millis, R. Mueller, and B. I. Shraiman, Phys. Rev. B 54, 5405 (1996).

[11] T. V. Ramakrishnan, Phil. Trans. Roy. Soc. Lond. A. 356, 41 (1998).

[12] D. M. Edwards, cond-mat/0201558; Adv. in Physics 51, 1259 (2002).

[13] Y. Tokura and Y. Tomioka, J. Magn. Magn. Mater. 200, 1 (1999). 
[14] K. Kanamori, J. Appl. Phys. 14S (1960).

[15] J. B. Goodenough, Phys. Rev. 100, 564 (1955).

[16] K. Kugel and D. I. Khomskii, JETP Lett. 15, 446 (1972); K. Kugel and D. Khomskii, Sov. Phys. JETP 37, 725 (1973); I. Khomskii and K. Kugel, Solid State Commun. 13, $763(1973)$.

[17] H. Kuwahara, et al., Science 270, 961 (1995).

[18] H. Kuwahara, T. Okuda, Y. Tomioka, A. Asamitsu and Y. Tokura, Phys. Rev. Lett 82, 4316 (1999).

[19] Y. Tomioka, A. Asamitsu, Y. Moritomo, H. Kuwahara and Y. Tokura, Phys. Rev. Lett 74, 5108 (1995).

[20] J. Hejtmanek et al., Phys. Rev. B 66, 014426 (2002).

[21] Y. Tomioka, et al., Phys. Rev. B 53, R1689 (1996).

[22] A. Urushibara, et al., Phys. Rev. B 51, 14103 (1995).

[23] M. Imada, A. Fujimori and Y. Tokura, Rev. Mod. Phys. 70, 1039 (1998).

[24] Andrzej M. Oles, Mario Cuoco and N. B. Perkins, cond-mat/0012013; (in Lectures on the Physics of Highly Correlated Electron Systems IV, edited by F. Mancini, AIP Conference Proceedings Vol. 527 (AIP, New York, 2000)).

[25] E. Muller-Hartmann and E. Dagotto, Phys. Rev. B 54, R6819 (1996).

[26] J. Hejtmanek et al., J. Appl. Phys. 918275 (2002).

[27] T. Akimoto et. al, Phys. Rev. B 57, R5594 (1998). 
[28] R. Kajimoto et. al., cond-mat/0110170; Phys. Rev. B 66, 180402(R) (2002).

[29] Z. Jirac et al., J. Magn. Magn. Mater. 15-18, 519 (1980).

[30] Z. Jirac et al., J. Magn. Magn. Mater. 53, 153 (1985).

[31] M. Tokunaga, et al., Phys. Rev. B 57, 5259 (1998).

[32] H. Kawano et. al., Phys. Rev. Lett. 78, 4253 (1997).

[33] S. Ishihara, I. Inoue and S. Maekawa, Physica C 263, 130 (1996); Phys. Rev. B 55, 8280 (1997).

[34] R. Kajimoto et. al, Phys. Rev. B 60, 9506 (1999).

[35] H. Yoshizawa et. al, Phys. Rev. B 58, R571 (1998).

[36] J. van den Brink and D. Khomskii, Phys. Rev. Lett 82, 1016 (1999).

[37] G. Venkateswara Pai, Phys. Rev. B 63, 064431 (2001).

[38] Tulika Maitra and A. Taraphder, Europhys. Lett. 59, 896 (2002).

[39] M. V. Zimmermann et. al., Phys. Rev. B 64, 195133 (2001).

[40] H. Kawano et. al., Physica B 241-243, 289 (1998).

[41] R. Maezono, S. Ishihara and N. Nagaosa, Phys. Rev. B. 57, R13993 (1998).

[42] R. Maezono, S. Ishihara and N. Nagaosa, Phys. Rev. B. 58, 11583 (1998).

[43] R. Maezono, et al., Mater. Science \& Engineering B, 63, 171 (1999). 58, 11583 (1998).

[44] D. Khomskii and G. A. Sawatzky, Solid State Commun. 102, 87 (1997). 
[45] J. van den Brink, G. Khaliullin and D. Khomskii, cond-mat/0206053; Phys. Rev. Lett 83, 5118 (1999).

[46] M. W. Long, Phil. Trans. Roy. Soc. Lond. A 356, 1493 (1998).

[47] E. D. Wollan and W. C. Koehler, Phys. Rev. 100, 545 (1955).

[48] P. G. Radaelli et al., Phys. Rev. Lett. 75, 4488 (1995).

[49] M. V. Zimmermann et. al., Phys. Rev. Lett. 83, 4872 (1999).

[50] A. Takahashi and H. Shiba, Eur. Phys. J. B 5, 413 (1998).

[51] A. Moreo, S. Yunoki and E. Dagotto, Science 283, 2034 (1999).

[52] M. Uehara, S. Mori, C. H. Chen and S. -W. Cheong, Nature 399, 560 (1999); V. Podzorov, M. Uehara, M. E. Greshenson, T. Y. Koo, S. -W. Cheong, Phys. Rev. B 61, R3784 (2000).

[53] Colossal Magnetoresiatance, Charge Ordering and Related Properties of Manganese Oxides, ed. C. N. R. Rao and B. Raveau, World Scientific (1998).

[54] Rahul Pandit, (unpublished).

[55] E. L. Nagaev, Physics of Magnetic semiconductors, Mir Publishers (Moscow, 1979); Sov. Phys. JETP. 30, 693 (1970); Physica B, 230-232, 816 (1997); Physics-Uspekhi 39, 781 (1996).

[56] M. Yu Kagan, D. I. Khomskii and M. V. Mostovoy, Eur. Phys. J. B 12217 (1999); M. Yu Kagan, K. I. Kugel and D. I. Khomskii, J. Exp. Theor. Phys. 93, 415 (2001).

[57] D. P. Arovas and F. Guinea, Phys. Rev. B 58, 9150 (1998); F. Guinea, G. GomezSantos and D. P. Arovas, Phys. Rev. B 62, 391 (2000). 
[58] J. L. Alonso, L. A. Ferdinandez, F. Guinea, V. Laliena and V. Martin-Mayor, Phys. Rev. B 63, 64416 (2000).

[59] E. Dagotto, J. Burgy and A. Moreo, cond-mat/0209689 and references therein.

[60] Ch. Renner, G. Aeppli, B. -G. Kim, Y-Ah Soh and S. -W. Cheong, Nature 416, 518 (2002).

[61] S. Yunoki et al., Phys. Rev. Lett. 80, 845 (1998); S. Yunoki, A. Moreo and E. Dagotto, Phys. Rev. Lett. 81, 5612 (1998).

[62] J. C. Loudon, N. D. Mathur and P. A. Midgley, cond-mat/0209436.

[63] J. C. Slater and G. F. Koster, Phys. Rev. 94, 1498 (1954).

[64] P. W. Anderson, Phys. Rev. 115, 2 (1959);

[65] T. Hotta, A. Malvezzi and E. Dagotto, Phys. Rev. B 62, 9432 (2000).

[66] H. Y. Hwang, et. al., Phys. Rev. Lett. 80, 1316 (1998). (1955).

[67] S. Misra, R. Pandit and S. Satpathy, Phys. Rev. B 56, 2316 (1997); J. Phys. Cond. Matter, 11, 8561 (1999).

[68] N. Furukawa, J. Phys. Soc. Jpn. 67, 2734 (1995); N. Furukawa in Ref. 1 and references therein.

[69] K. Kubo and N. Ohata, J. Phys. Soc. Jpn. 33, 21 (1972).

[70] S. Satpathy et. al, Phys. Rev. Lett. 76, 960 (1996).

[71] A. Maignan, C. Martin, F. Damay and B. Raveau, Phys. Rev. B 58, 2758 (1998); R. Mahendiran et. al., Phys. Rev. B 62, 11644 (2000). 
[72] The phase identified as canted A-phase by BK is actually a canted G-phase. This phase should be contrasted with the A-phase close to $x=0.5$ end which is orbitally ordered.

[73] L. F. Feiner and A. M Oles, Phys. Rev. B 59, 3295 (1999).

[74] L. Sheng and C. S. Ting, cond-mat/9812374.

[75] G. Jackeli, N. B. Perkins and N. M. Plakida, Phys. Rev. B 62, 372 (2001).

[76] C. Castellani et. al., Phys. Rev. B 18, 4945 (1978); S. Inagaki, J. Phys. Soc. Jpn. 39 596 (1975).

[77] The changes in $\theta_{x y, z}$ for small electron-doping are small and depend weakly on $y$. Furthermore, the change in SE energy depends quadratically on the changes in $\theta_{x y, z}$.

[78] K. H. Ahn et al., J. Appl. Phys. 81, 5505 (1997).

[79] J. Blasco, et al., Phys. Rev. B 55, 8905 (1997).

[80] V. Cerovsky, S. D. Mahanti, T. A. Kaplan and A. Taraphder, Phys. Rev. B 59, 13977 (1999).

[81] Z. Jirac et al., cond-mat/0212517.

[82] R. D. Shannon, Acta Crystallogr. Sec. A, 32, 751 (1976).

[83] J. Dho et al., Phys. Rev. Lett. 89, 027202 (2002).

[84] The CE-phase close to $x \leq 0.5$ appears in some cases in a narrow region for the intermediate bandwidth systems $[28,27,32]$. For $\left(\mathrm{La}_{0.5} \mathrm{Nd}_{0.5}\right)_{1-x} \mathrm{Sr}_{x} \mathrm{MnO}_{3}$, the region $(z \simeq 0.4$ and $x \geq 0.5$ ) shows no charge ordering (see fig. 2 in the present paper $\&$ fig. $4 \mathrm{in}[27])$.

[85] The numerical results converged by the system size $64 \times 64 \times 64$. 
[86] R. Maezono, S. Ishihara and N. Nagaosa, Phys. Rev. B. 57, R13993 (1998).

[87] R. Maezono, S. Ishihara and N. Nagaosa, Phys. Rev. B. 58, 11583 (1998).

[88] In the choice of $\mathrm{J}_{H} \mathrm{~S}_{0}$ in eqn.(3), there is an additional factor of 2 in ref.[37].

[89] K. Kugel and D. Khomskii, Sov. Phys. JETP 37, 725 (1973).

[90] Q. Yuan, T. Yamamoto and P. Thalmeier, Phys. Rev. B. 62, 12696 (2000).

[91] Tulika Maitra and A. Taraphder, (unpublished).

[92] Tulika Maitra and A. Taraphder, (unpublished).

[93] Unjong Yu, Yookyung Jo and B. I. Min, cond-mat/0209230.

[94] J. L. Alonso, L. A. Fernandez, F. Guinea, V. Laliena and V. Martin-Mayor, condmat/0111244.

[95] C. D. Lin et al., Phys. Rev. B. 62, 15096 (2000).

[96] G. V. Pai, private communication. 


\section{Figure captions}

Fig. 1 Schematic phase diagram in the band-width versus hole concentration in the series of three dimensional manganites after Kajimoto et al. [28]. The labels represent different magnetic phases explained in the text. $\mathrm{C}_{x} \mathrm{E}_{1-x}$ stands for an incommensurate chargeordered and CE-type spin ordered phase.

Fig. 2 Phase diagram in $z$ versus hole concentration plane for $\left(\mathrm{La}_{1-z} \mathrm{Nd}_{z}\right)_{1-x} \mathrm{Sr}_{x} \mathrm{MnO}_{3}$ after Akimoto et al.[27]. The effective band-width decreses as $z$ increases. COI stands for charge-ordered insulating phase.

Fig. 3. (a) Band dispersions in the A-phase along the different symmetry directions of a cubic Brillouin zone. Note the lack of dispersion along $z$-direction. In (b) is shown the dispersion in the magnetically isotropic state where the upper band now disperses along $\Gamma-z$ direction.

Fig. 4. Ground state energy of different magnetic phases versus hole-concentration $x>0.5$ close to the respective transitions (F-phase to A-phase in (a), A-C in (b) and C-G in (c)) for $J_{H} S_{0}=16$ and $J_{A F} S_{0}^{2}=0.05$. All energies are measured in units of hopping $t$.

Fig. 5. Magnetic phase diagram in doping $(x)-\mathrm{J}_{H} \mathrm{~S}_{0}$ plane with $U^{\prime}=0$.

Fig. 6. Canting of the angles $\theta_{x y}$ and $\theta z$ in degrees (a) as a function of $\mathrm{J}_{H} S_{0}$ for $\mathrm{J}_{A F} S_{0}^{2}=$ 0.04 (solid line),0.05 (dotted line) and 0.06 (dashed line) and (b) $\theta_{x y}$ versus $\mathrm{J}_{A F} S_{0}^{2}$ at $\mathrm{J}_{H} S_{0}=10$.

Fig. 7. Same as Fig. 4, in the presence of onsite inter-orbital Coulomb interaction $U^{\prime}$.

Fig. 8. Magnetic phase diagram in doping $(x)-J_{H} S_{0}$ plane for different $U^{\prime}$. Note the gradual shrinking of the F-phase in the region $x>0.5$. For low $U^{\prime}$ the size of the A-phase 
remains unaffected but at larger $U^{\prime}$ it rapidly shrinks. The C-phase grows a bit while the G-phase remains nearly unaffected.

Fig. 9. Magnetic phase diagram in doping $(x)-\mathrm{J}_{A F} \mathrm{~S}_{0}^{2}$ plane with $U^{\prime}=0$ and 8.

Fig. 10. Orbital densities as a function of doping $x$ for three values of $U^{\prime}=0,4,8$. The filled symbols are for $d_{z^{2}}$ and open symbols for $d_{x^{2}-y^{2}}$ orbitals. The vertical dotted lines represent the boundary between A- and C-phases for different $U^{\prime}$. We choose $\mathrm{J}_{H} S_{0}=5$ here in order to have stable A- and C-phases for a reasonable range of $x$ (see figs. 5 and 8) for all three $U^{\prime}$ values. $\mathrm{J}_{A F} S_{0}^{2}$ was kept at 0.05 .

Fig. 11. Orbital density versus $U^{\prime}$ in (a) A-phase at $x=0.5$ and (b) C-phase at $x=0.65$. The dotted lines are for $d_{z^{2}}$ and solid lines are for $d_{x^{2}-y^{2}}$ orbitals. $\mathrm{J}_{H} S_{0}$ and $\mathrm{J}_{A F} S_{0}^{2}$ were same as in fig. 10 .

Fig. 12. Magnetic phase diagram in doping $(x)-\mathrm{J}_{H} \mathrm{~S}_{0}$ plane for three different $V$. The F-CO region gets wider with increasing $V$. The $\mathrm{F}-\mathrm{CO}$ to $\mathrm{C}$ transition is first order and the inhomogeneous boundary region is shown with shading. The other transitions are continuos as in figs. 5 and 8.

Fig. 13. Magnetic phase diagram in doping $(x)-\mathrm{J}_{H} \mathrm{~S}_{0}$ plane for finite $V$ at two different values of $U^{\prime}$. Note that on changing $U^{\prime}$ the trend follows that in figs. 5 and 8. The F-CO to C transition is not shaded here to show the effect of changing $U^{\prime}$.

Fig. 14. The charge-order parameter versus hole concentration for $\mathrm{J}_{A F} S_{0}^{2}=0.05$.

Fig. 15. The charge-order parameter versus near-neighbour Coulomb interaction strength for two different hole concentrations. The transition $\mathrm{F}$ to $\mathrm{F}-\mathrm{CO}$ as a function of $V$ is continuous. 
Fig. 16. Summary of the general trend observed in the various phase diagrams (for $V=0$ ). Note the trend with increasing $U^{\prime}$ follows closely that of fig. 1 with decreasing bandwidth. 


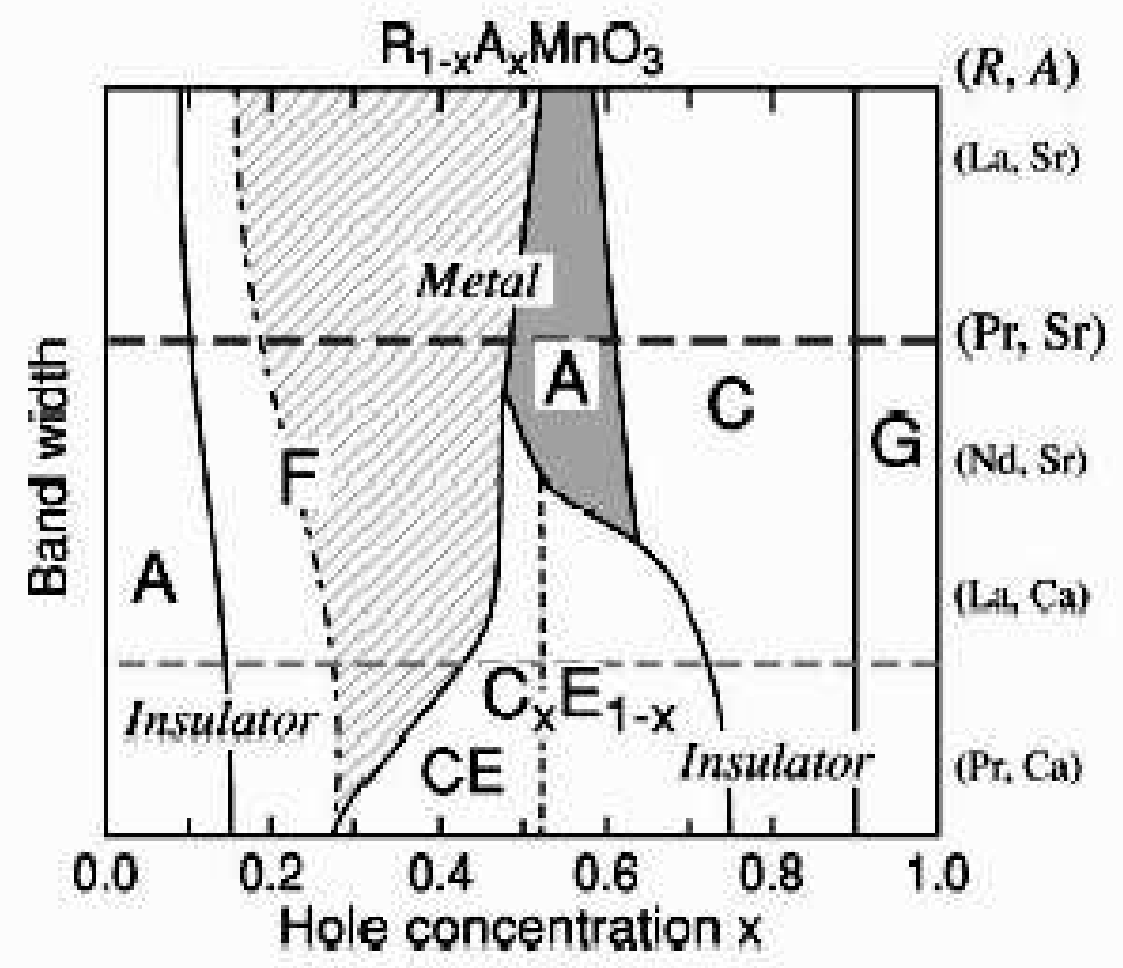




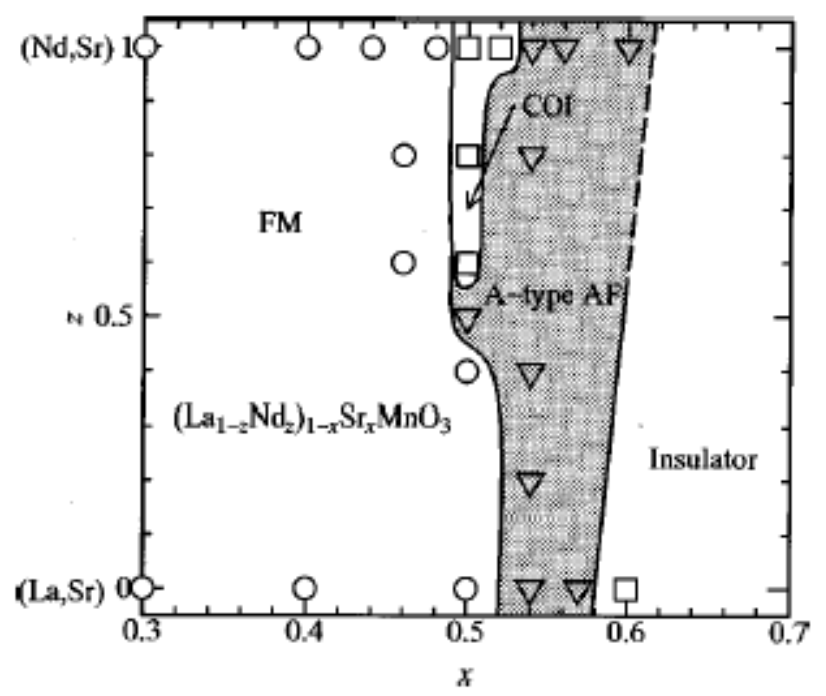




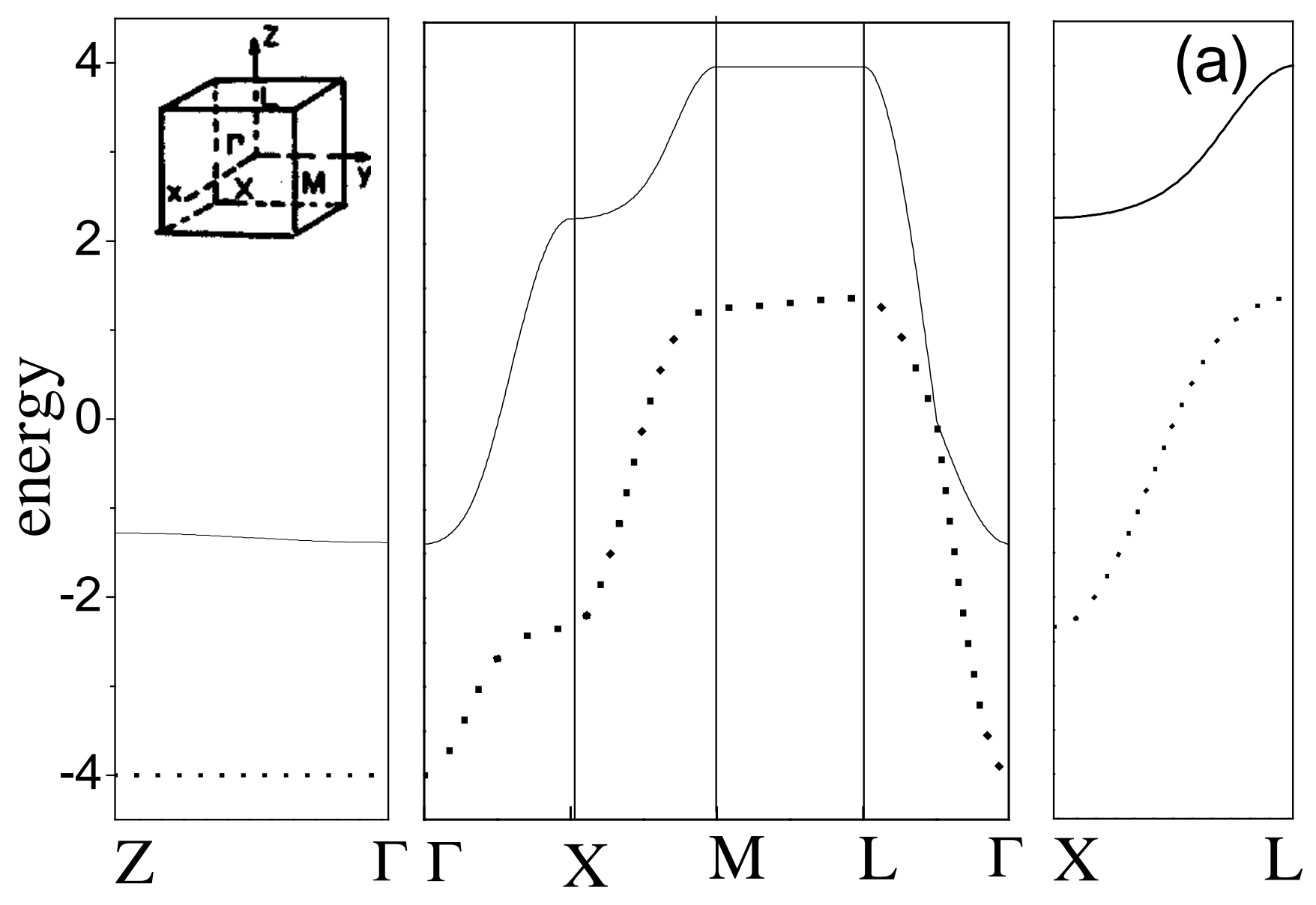




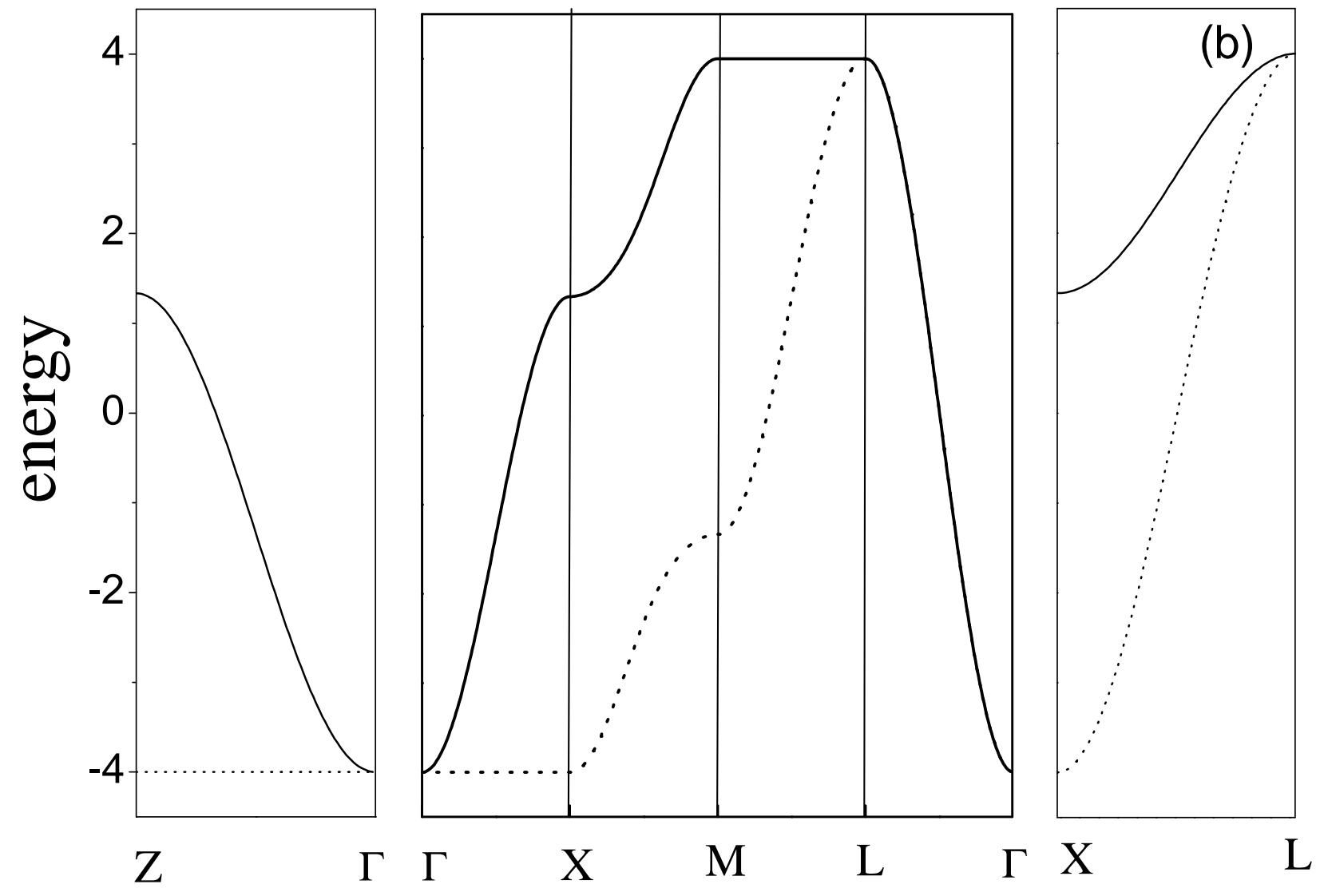



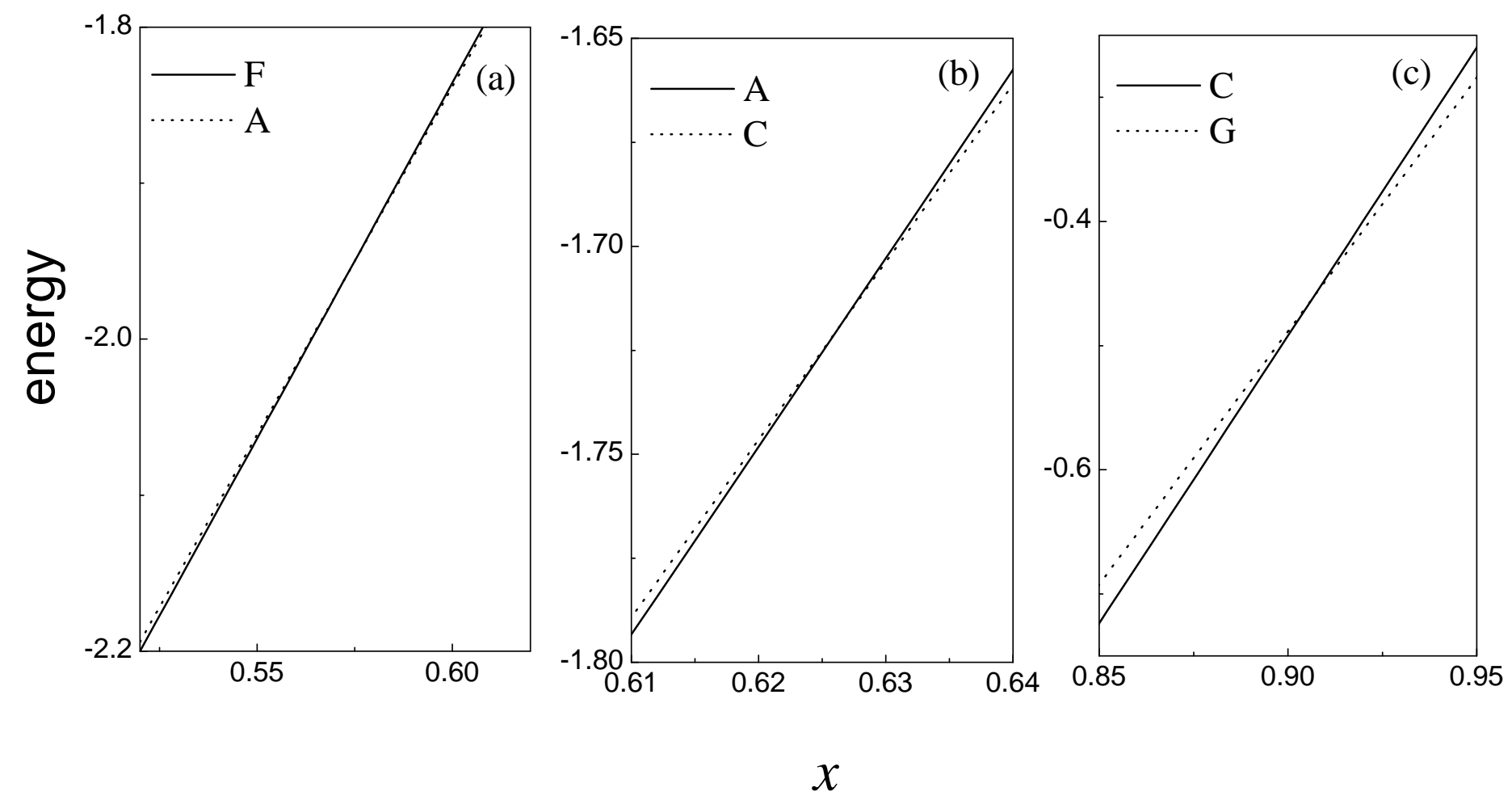


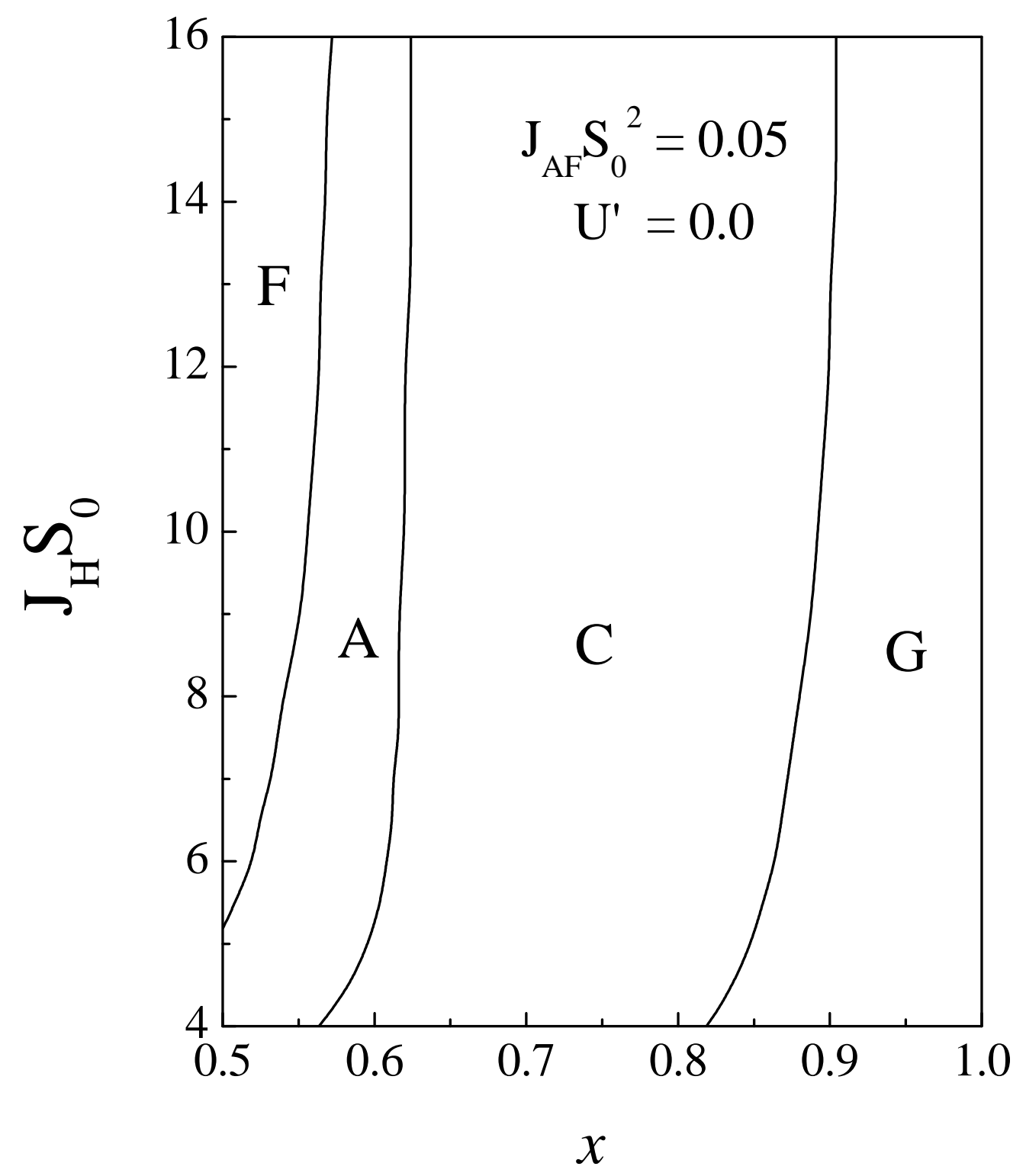




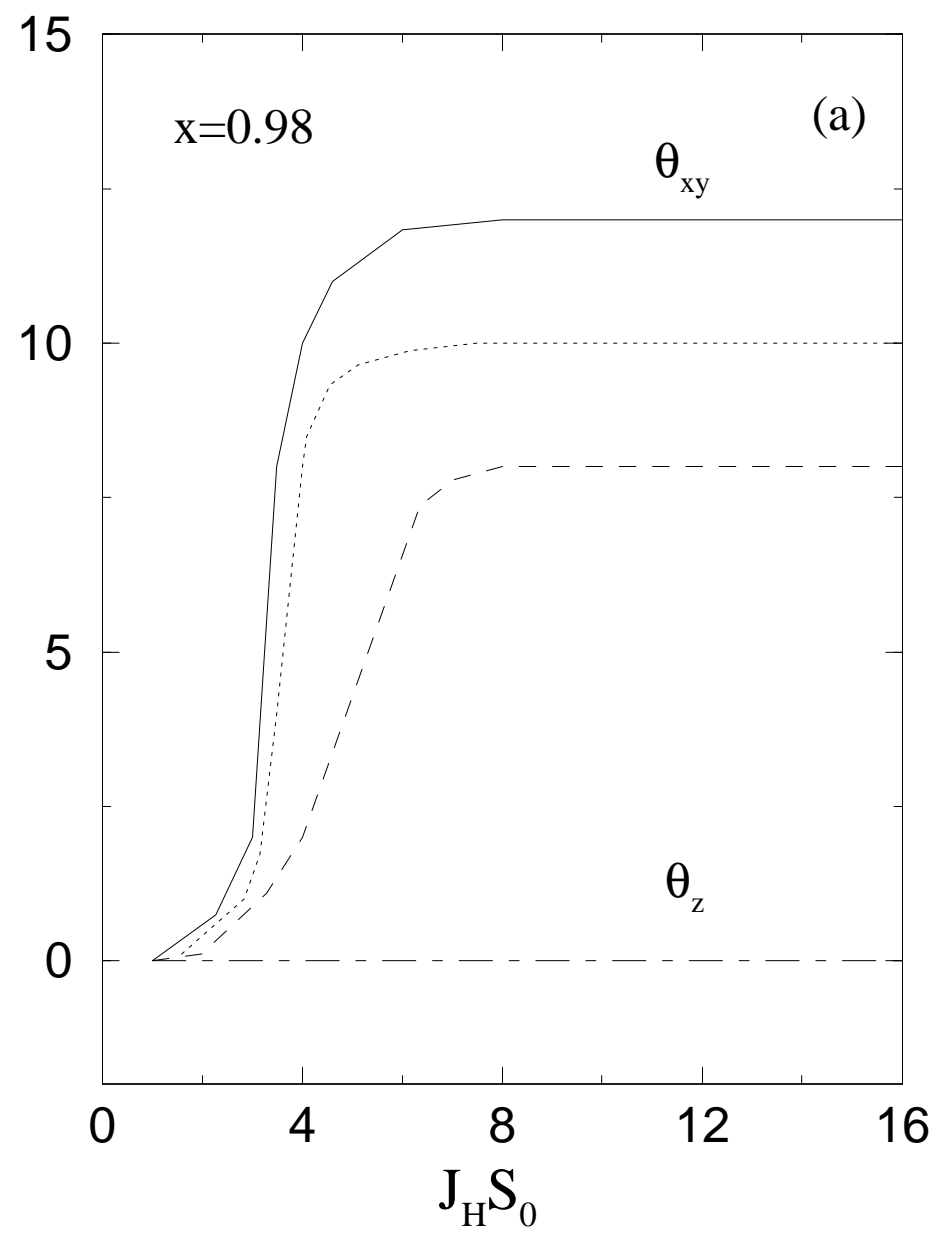




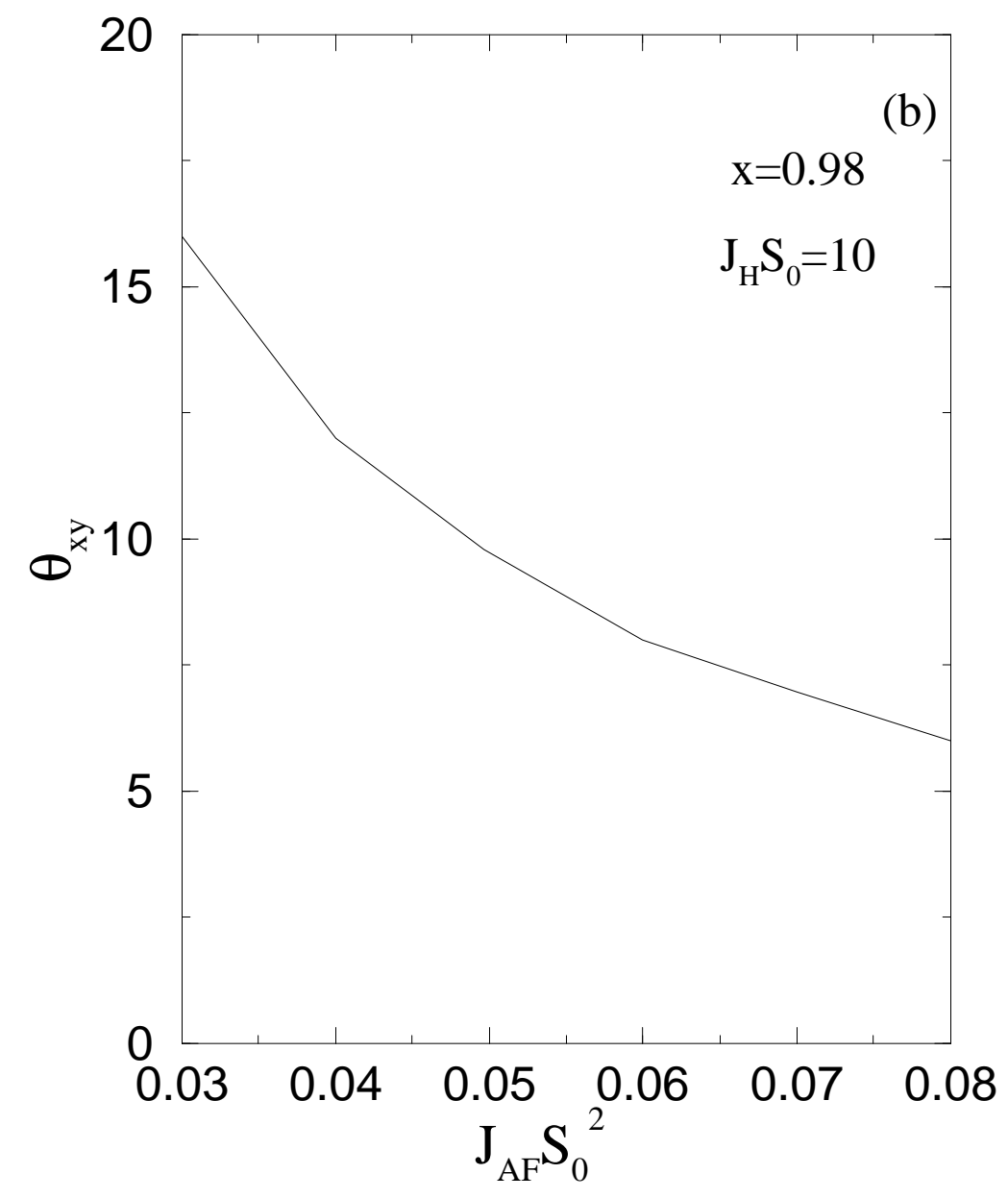



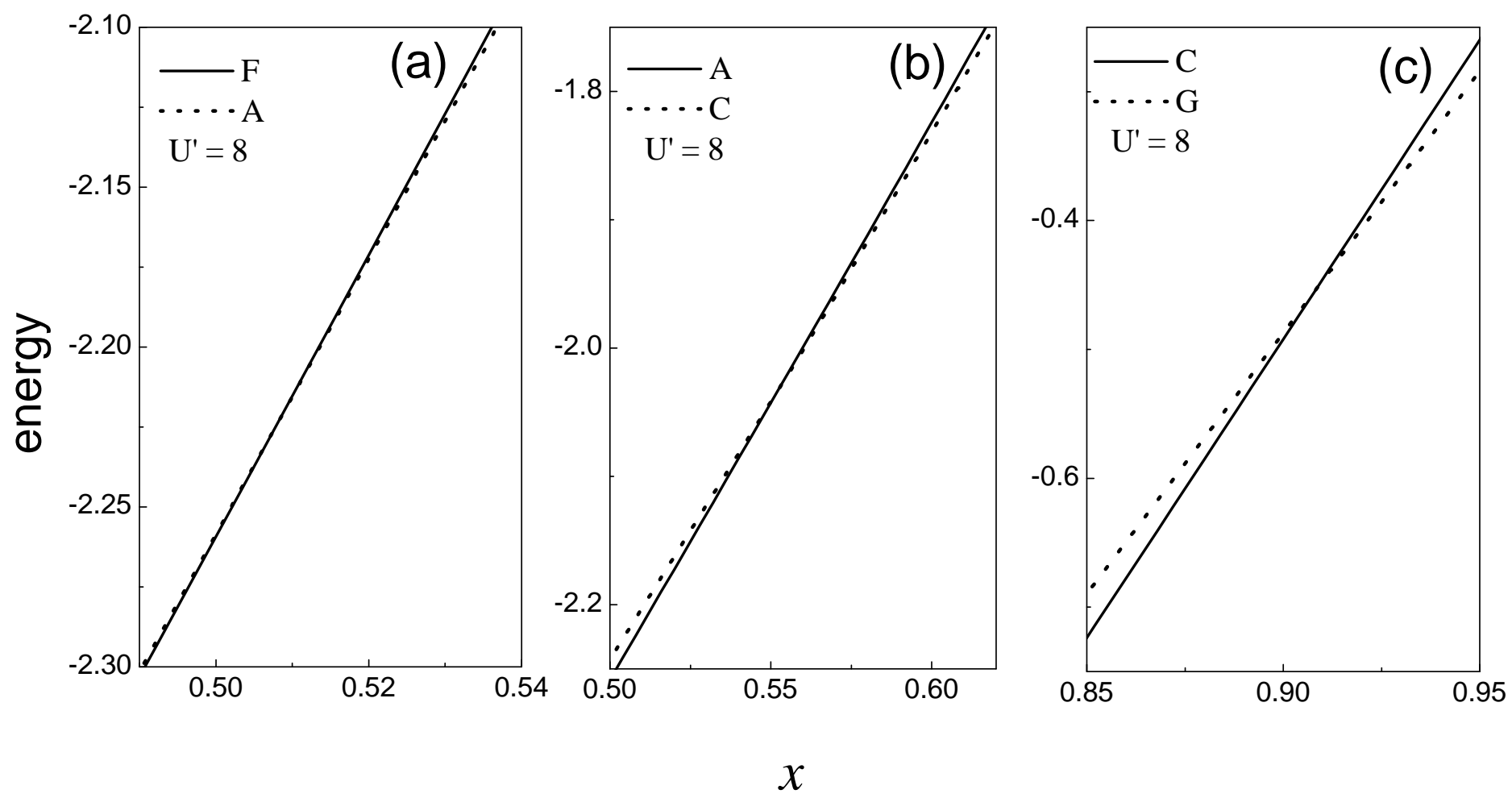

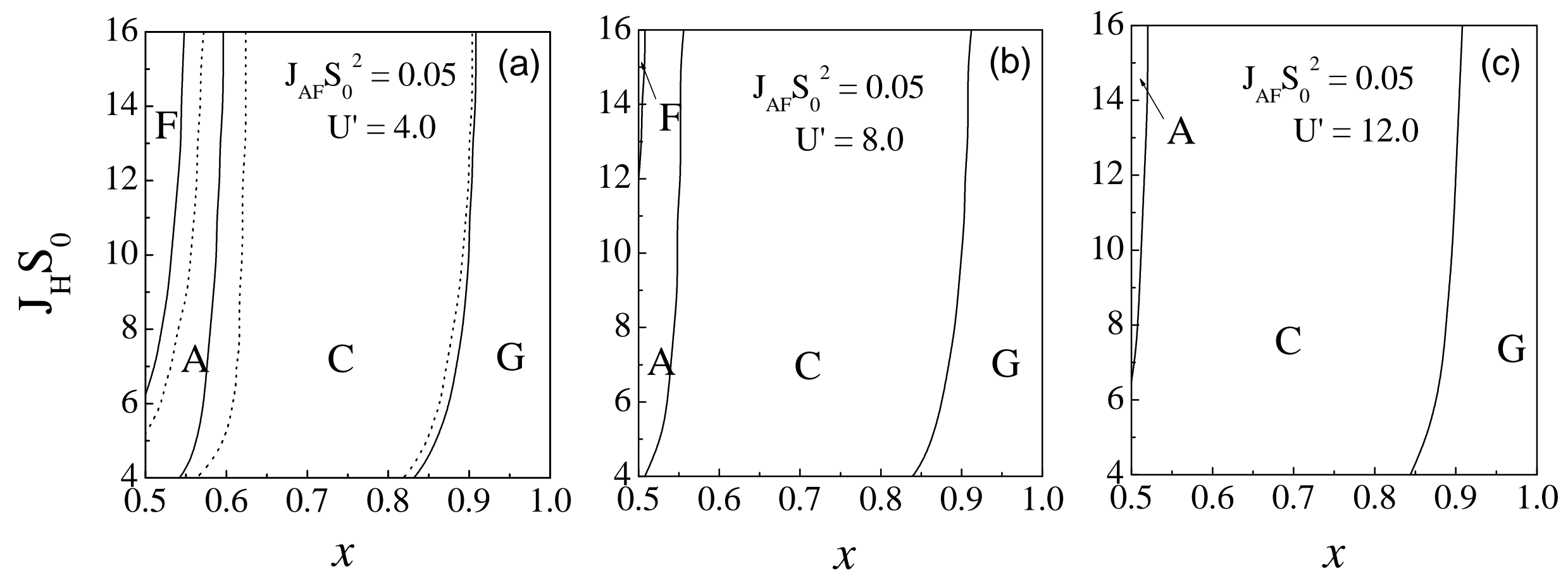


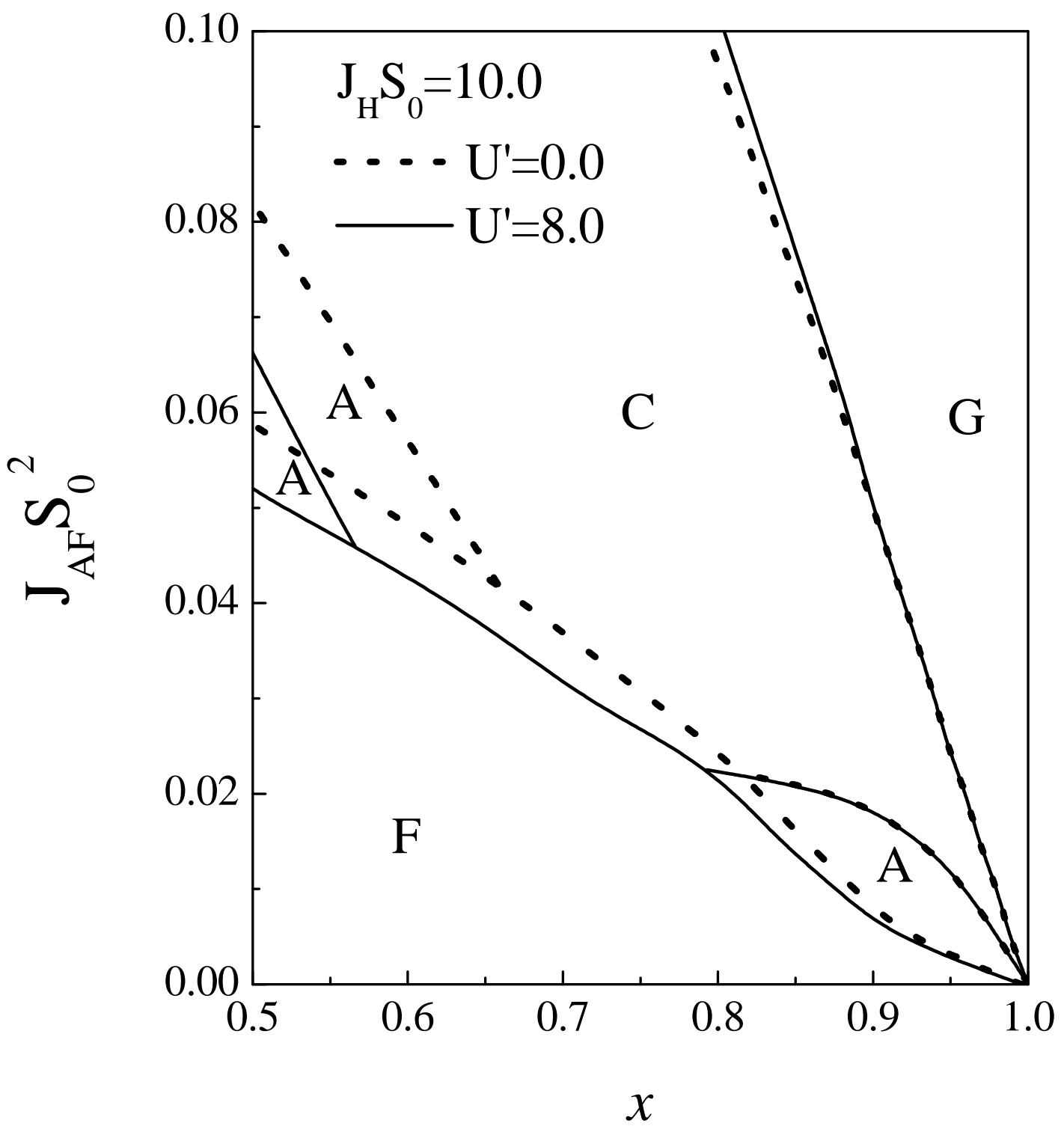




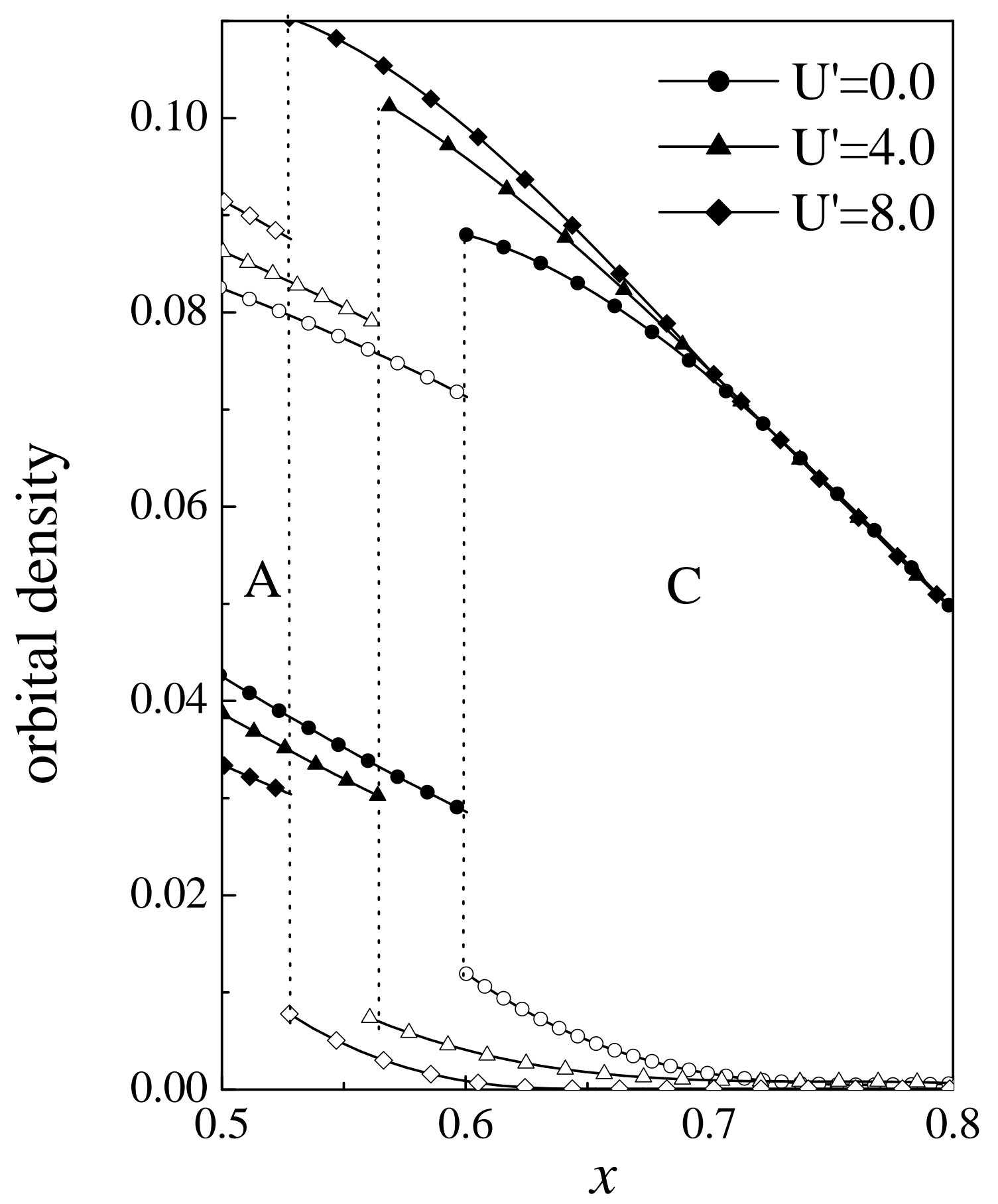




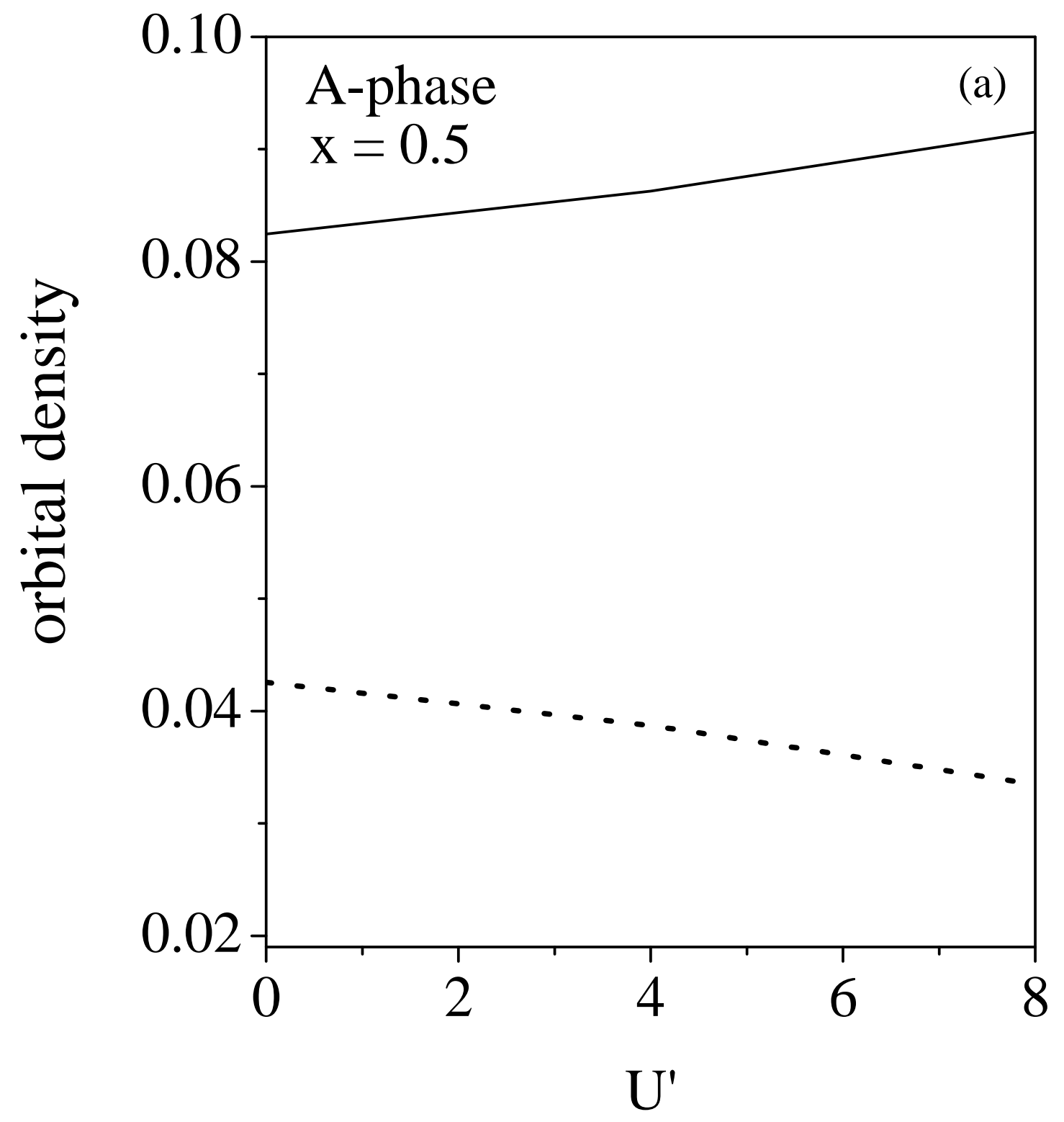




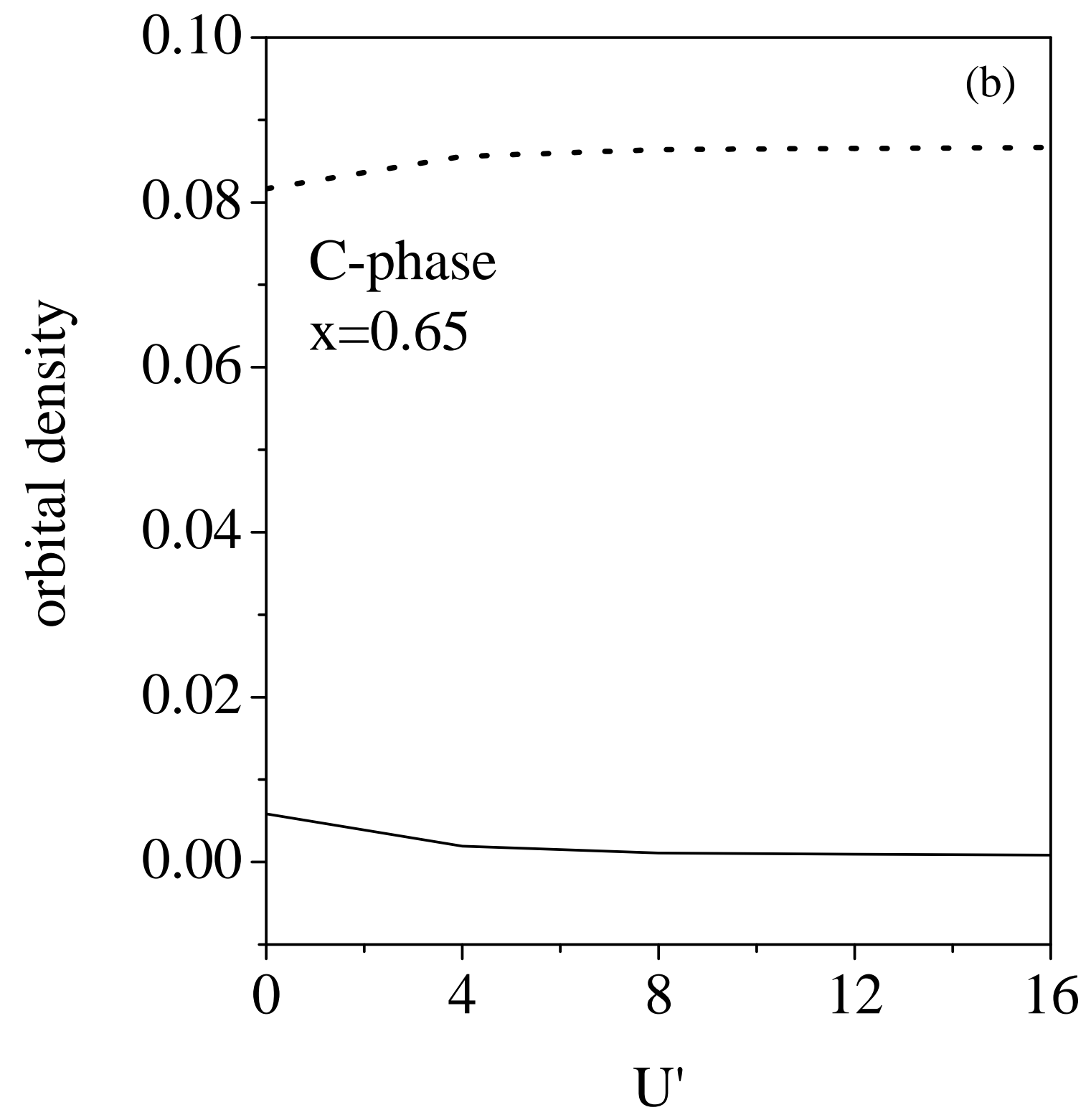



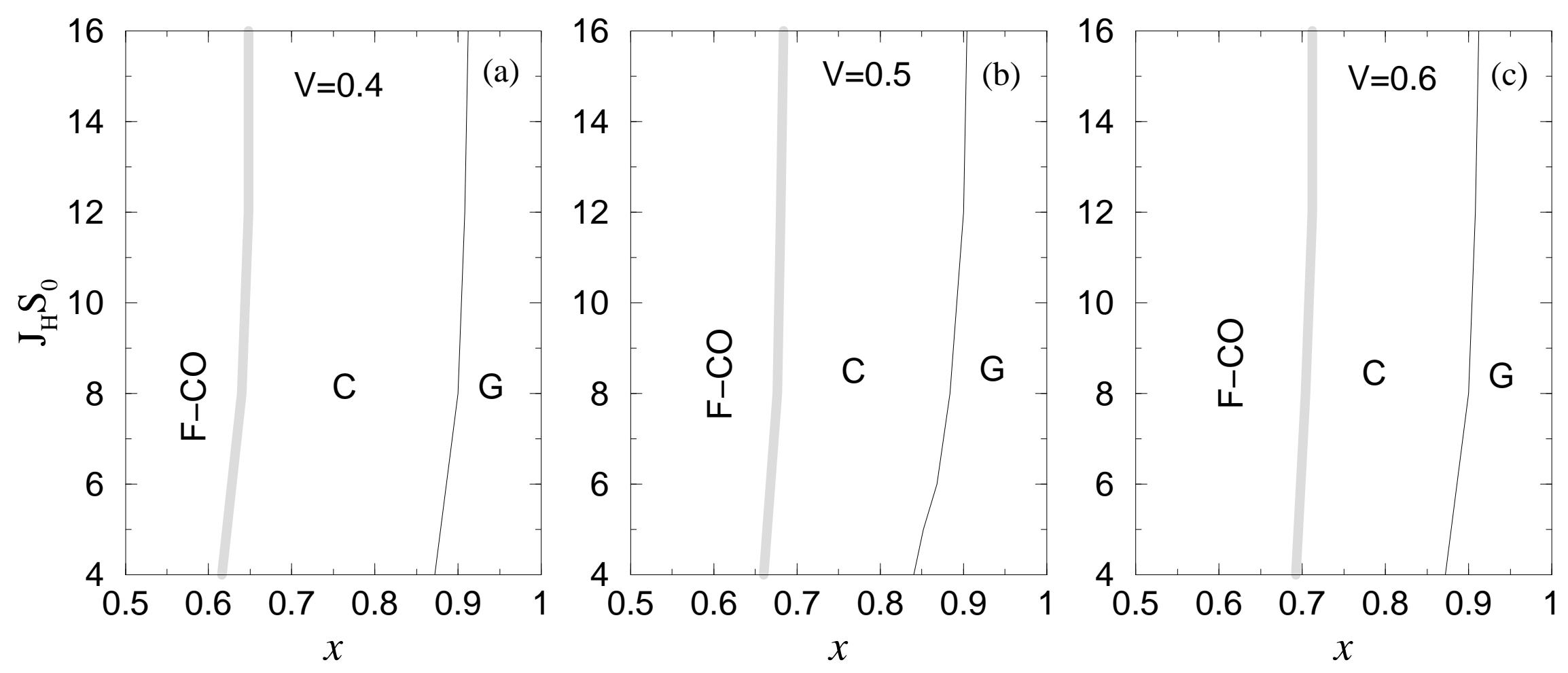


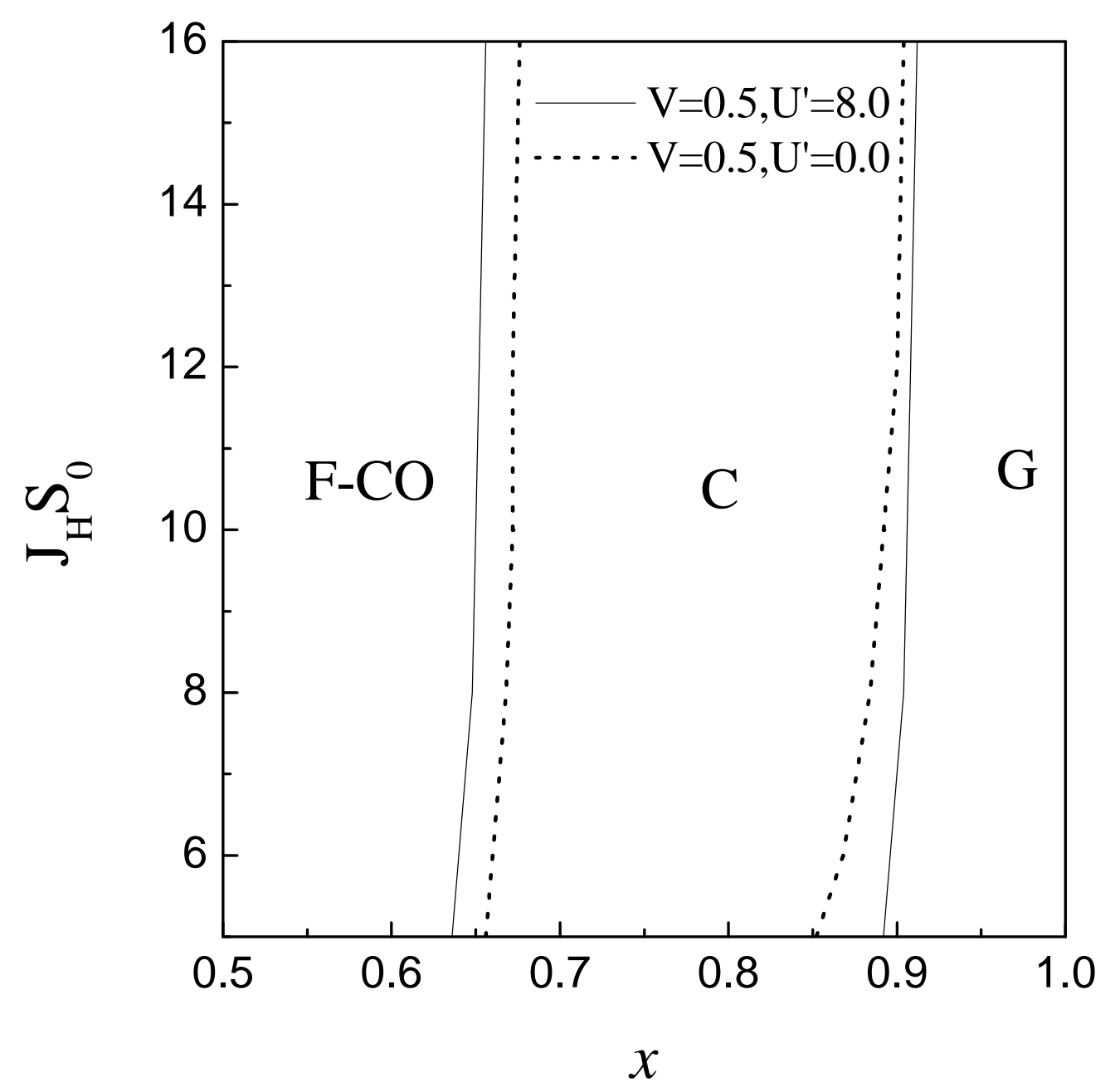




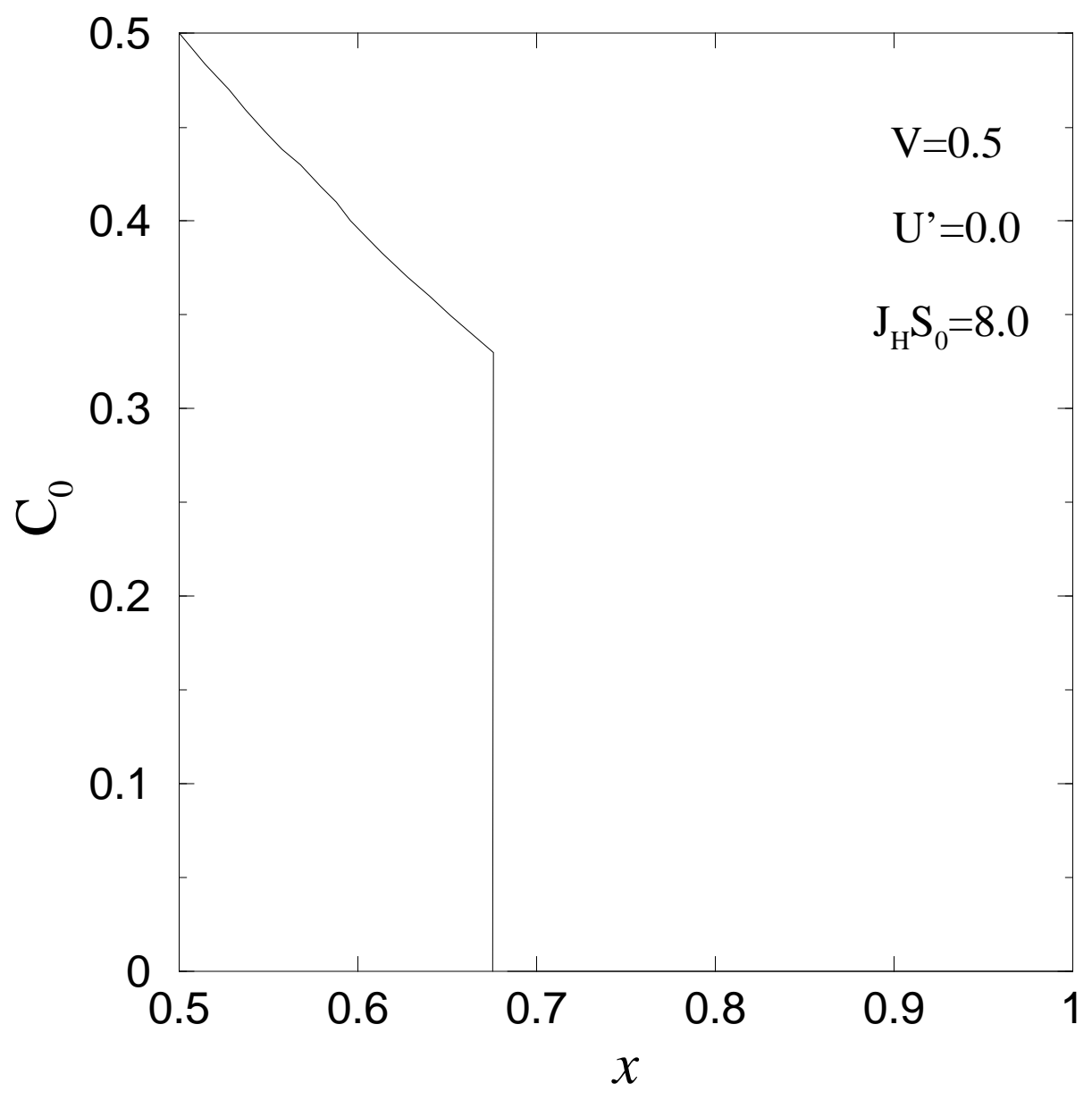




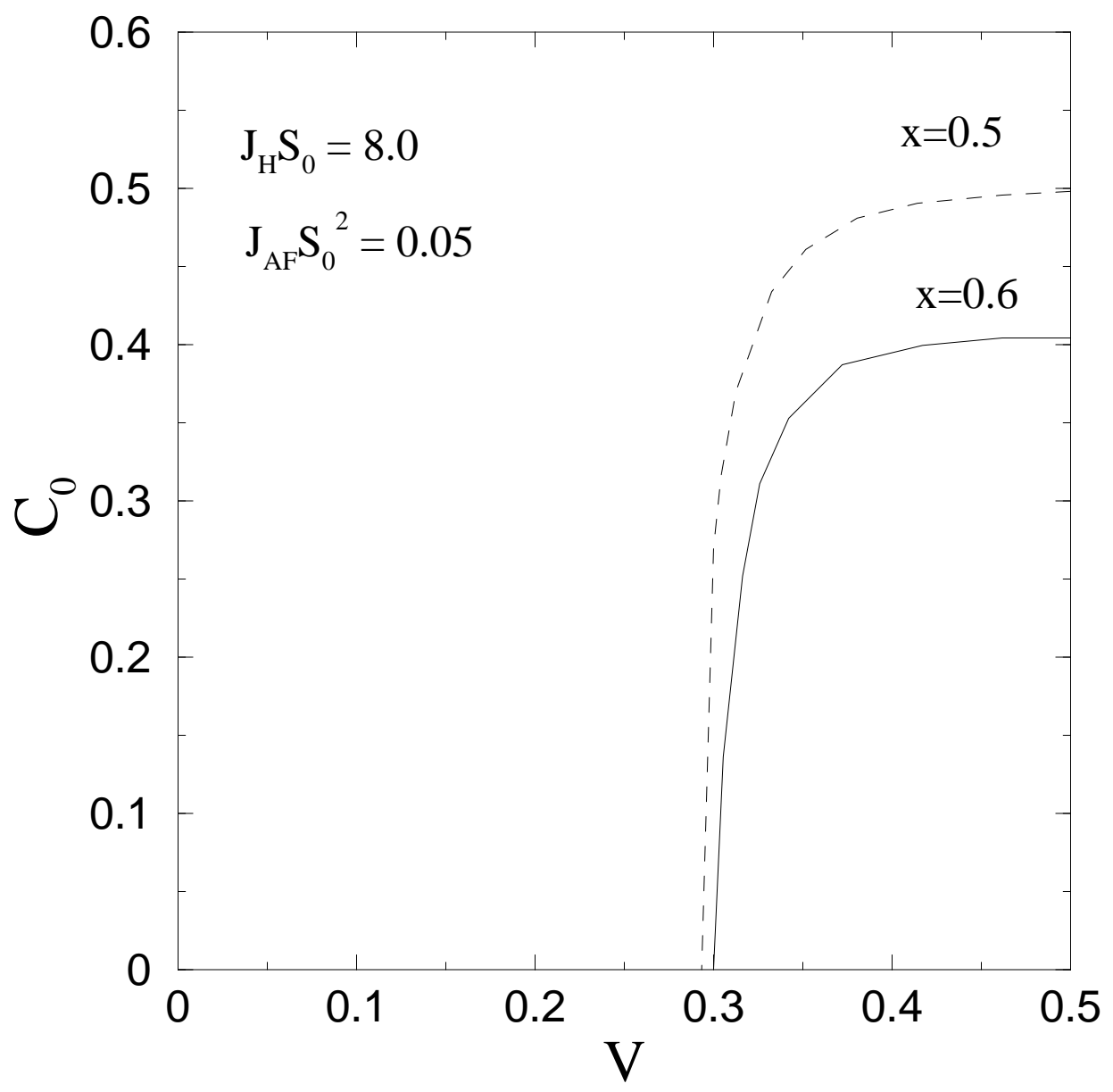




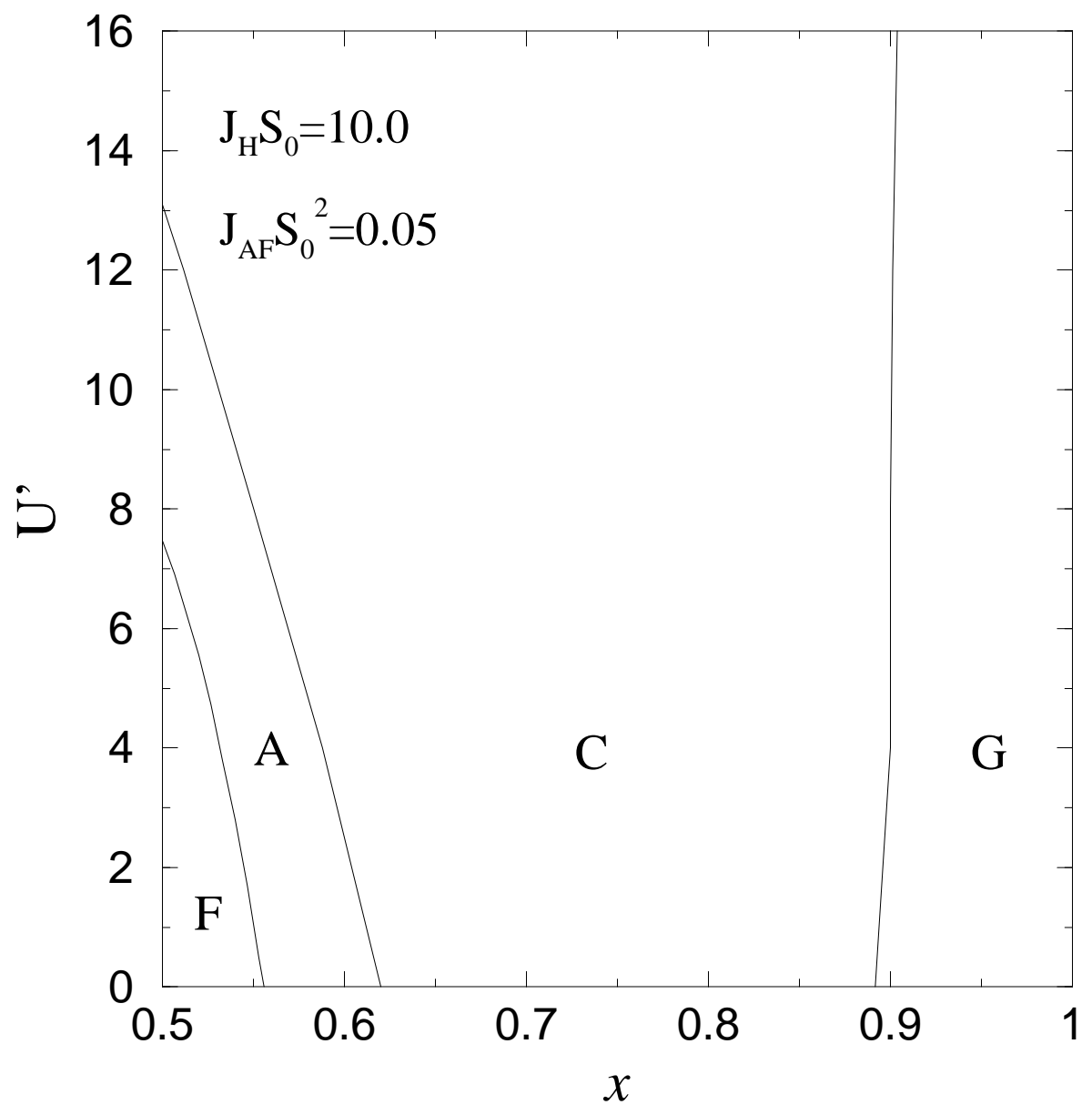

